\title{
A causal role for the parietal cortex in ambiguity computations in humans
}

Gabriela Valdebenito-0yarzo ( $\sim$ gvaldebenitoo@udd.cl)

Universidad del Desarrollo

María Paz Martínez-Molina1 ( $\nabla$ mariamartinezmo@udd.cl)

Universidad del Desarrollo

Patricia Soto-Icaza ( $\triangle$ patriciasoto@udd.cl )

Universidad del Desarrollo https://orcid.org/0000-0002-3092-7195

Francisco Zamorano ( $\square$ fzamorano@udd.cl )

Universidad del Desarrollo

Alejandra Figueroa-Vargas ( $\square$ amfigueroa@udd.cl)

Universidad del Desarrollo

Josefina Larraín-Valenzuela ( $\sim$ josefinalarrain@udd.cl )

Universidad del Desarrollo

Ximena Stecher ( $\nabla$ xstecher@gmail.com )

Clinica Alemana

César Salinas ( $\square$ csalinasg@gmail.com )

Clinica Alemana

Antonie Valero-Cabré ( $\square$ antoni.valerocabre@icm-institute.org )

Institut du Cerveau et de la Moelle Epinière (ICM)

Rafael Polania ( $\nabla$ rafael.polania@hest.ethz.ch )

ETH Zurich https://orcid.org/0000-0002-6176-6806

Pablo Billeke ( $\sim$ pbilleke@udd.cl)

Universidad del Desarrollo https://orcid.org/0000-0003-4261-0678

Julian Bustin

University of grenoble

Article

Keywords: Decision-making, Risk, Ambiguity, Uncertainty, Parietal cortex, Intraparietal sulcus, Prediction error, EEG, TMS, fMRI

DOl: https://doi.org/10.21203/rs.3.rs-1362283/v2 
License: (c) (i) This work is licensed under a Creative Commons Attribution 4.0 International License. Read Full License

Additional Declarations: There is NO Competing Interest. 


\section{Full Title \\ A causal role for the parietal cortex in ambiguity computations in humans}

\section{Short Title \\ Parietal cortex computes ambiguity in humans}

\section{Authors}

Gabriela Valdebenito-Oyarzo ${ }^{1}$, María Paz Martínez-Molina ${ }^{1}$, Patricia Soto-Icaza ${ }^{1}$, Francisco Zamorano ${ }^{2}$, Alejandra Figueroa-Vargas ${ }^{1}$, Josefina Larraín-Valenzuela ${ }^{1}$, Ximena Stecher ${ }^{2}$, César Salinas ${ }^{2}$, Julien Bastin $^{3}$, Antonie Valero-Cabré, ${ }^{4,5,6}$, Rafael Polania ${ }^{7} \&$ Pablo Billeke*1.

\section{Affiliations}

${ }^{1}$ Laboratorio de Neurociencia Social y Neuromodulación, Centro de Investigación en Complejidad Social, (neuroCICS), Facultad de Gobierno, Universidad del Desarrollo, Santiago, Chile.

${ }^{2}$ Departamento de Imágenes, Clínica Alemana de Santiago, Chile.

${ }^{3}$ Univ. Grenoble Alpes, Inserm, U1216, Grenoble Institut Neurosciences, 38000 Grenoble, France

${ }^{4}$ Cognitive Neuroscience and Information Technology Research Program, Open University of Catalonia (UOC), Barcelona, Spain.

${ }^{5}$ Laboratory for Cerebral Dynamics Plasticity and Rehabilitation, Boston University, School of Medicine, Boston, MA, USA.

${ }^{6}$ Causal Dynamics, Plasticity and Rehabilitation Group, FRONTLAB team, Institut du Cerveau et de la Moelle Epinière (ICM), CNRS UMR 7225, INSERM U 1127 and Sorbonne Université, Paris, France.

${ }^{7}$ Decision Neuroscience Lab, Department of Health Sciences and Technology, ETH Zurich, Zurich, Switzerland.

* Corresponding author: pbilleke@udd.cl

\section{Teaser}

The parietal cortex is involved in computational processes that underestimate uncertainty during decision-making under ambiguity. 


\section{Highlights}

- Humans assign unknown probabilities to outcomes during decision-making under ambiguity, increasing the uncertainty of decision-making.

- Parietal cortex activity correlates with ambiguity computations associated with underestimating uncertainty during decision-making.

- Evaluating outcomes under ambiguity increases connectivity between the parietal and midcingulate cortex, which in turn senses prediction errors.

- Perturbation of parietal activity increases the assignment of unknown probabilities and reduces midcingulate cortex oscillations associated with prediction error.

\section{Abstract \\ $(146 / 160$ word max $)$}

Humans often face the challenge of making decisions between ambiguous options. The level of ambiguity in decision-making has been linked to activity in the parietal cortex, but its exact computational role remains elusive. To test the hypothesis that the parietal cortex plays a causal role in computing ambiguous probabilities, we conducted consecutive fMRI and TMS-EEG studies. We found that participants assigned unknown probabilities to objective probabilities, elevating the uncertainty of their decisions. Parietal cortex activity correlated with both the objective degree of ambiguity and a process that underestimates the uncertainty during decision-making. Conversely, the midcingulate cortex encodes prediction errors and increases its connectivity with the parietal cortex during outcome processing. Disruption of the parietal activity increased the uncertainty evaluation of the options, decreasing cingulate cortex oscillations during outcome evaluation. These results provide evidence for a causal role of the parietal cortex in computing uncertainty during ambiguous decisions made by humans.

\section{INTRODUCTION}

Making decisions in situations where the availability of outcomes is uncertain is a prevalent challenge in daily life. For instance, when visiting a city for the first time, selecting a restaurant that serves the desired pizza can pose a dilemma. Making choices based on incomplete information about the availability of potential outcomes is a common phenomenon among humans and other animals. These circumstances exhibit ambiguity, a type of uncertainty that may vary depending on the individual's knowledge of the environment (1). Despite the common occurrence of ambiguous scenarios in ecological settings, organisms generally avoid such situations, a phenomenon known as ambiguity aversion (2-5). Nevertheless, life often presents situations where ambiguity cannot be avoided, and individuals must make decisions based solely on available information. How humans make decisions when it is impossible to avoid ambiguity and what neurobiological mechanisms underlie such computations remain unclear. 
When evaluating options and making decisions, individuals consider available information, such as the probability of outcomes and their associated rewards. This evaluation process appears to rely on a neural network consisting of the ventromedial prefrontal cortex, orbitofrontal cortex, and ventral striatum (6-8). Moreover, the intraparietal sulcus (IPS) and dorsal posterior parietal cortex (PPC) (911) have been implicated in the perception and comparison of varying levels of uncertainty during decision-making. Additionally, research has shown a correlation between activity in the frontal and parietal areas and the degree of uncertainty and the updating processing that reduces uncertainty (10, 12). Furthermore, recent studies in non-human primates have identified a crucial role for the parietal cortex in encoding the expected reduction of uncertainty during perceptual decision-making processes (13). Activity in the parietal cortex has been associated with surprise signaling in human studies, but its connection to decision-making is unclear (14). As a result, the specific role of the parietal cortex during decision-making under ambiguity and its causal contributions to these processes in humans remains elusive.

Evaluating the outcomes of our decisions is another essential aspect of decision-making behavior, as it enables us to learn and update our knowledge of the environment. Upon making a decision, individuals assess whether the outcome matches their expectations, resulting in a prediction error signal that can be detected in various brain regions, even in situations where no explicit learning occurs (15-18). After a decision, research has revealed sustained connectivity between the parietal region and prefrontal cortex, which influences future decision-making $(19,20)$. Unexpected outcomes in uncertain situations have been linked to prefrontal activity, as indicated by fMRI studies (11) and oscillatory activity from EEG recordings $(21,22)$. A large body of work has shown a correlation between frontal delta and theta activity and prediction errors in uncertain situations $(21,23-25)$. Despite the well-established role of the connectivity between parietal and frontal regions in decisionmaking and value representations $(20,26)$, the impact of the parietal activity on frontal prediction error signal arising from decisions under ambiguity is still unclear.

In this study, we aimed to investigate the hypothesis that the parietal cortex has a causal role in the process of valuing ambiguous options when making decisions. Previous research has utilized the dichotomy between ambiguous and non-ambiguous options to analyze ambiguity (27); however, this approach does not accurately depict real-life decision-making situations. To better understand the unique aspects of ambiguity computation in daily life decisions, we designed an experiment incorporating behavioral modeling and fMRI information to be tested in a subsequent TMS-EEG study. Our results showed that participants assigned some proportion of the unknown probability to objective, known probability during decision-making, increasing the uncertainty of their decisions. This process was linked to parietal activity during the decision period and midcingulate cortex (MCC) activity during feedback. Furthermore, TMS disruption of parietal activity led to an increase in unknown probability assignment and a reduction in delta activity in the MCC during feedback, confirming a causal role of the parietal cortex in the computation of ambiguous information of the options during decision-making. 


\section{RESULTS}

\section{Behavior}

In the designed task, participants were required to choose between two options. Each option was associated with a different probability of being rewarded and a varying magnitude of reward, as depicted in Figure 1. In half of the trials (ambiguity condition), the actual probabilities were partially concealed, resulting in varying degrees of ambiguity, ranging from $40 \%$ to $80 \%$ occlusion $P_{a}=$ $\{0,0.4,0.5,0.6,0.7,0.8\})$. This manipulation aimed to examine the effect of ambiguity on decisionmaking behavior. The probabilities and the rewards were misaligned, meaning that the option with the highest objective probability $\left(P_{o b s}\right)$ was not associated with the highest reward. This feature enabled us to calculate the rate at which participants chose the option with the highest probability despite offering a lower reward. Our results revealed that participants favored the option with the highest objective probability without ambiguity (no-ambiguity condition, rate $=0.65$; Wilcoxon test, $\mathrm{n}=38, \mathrm{p}=3 \mathrm{e}-5$, logit mixed model, intercept: beta $=1.17$, s.e. $=0.18, \mathrm{z}$-value $=6.1, \mathrm{p}=6.8 \mathrm{e}-10, \mathrm{~d} . \mathrm{f} .=1444$; Figure 2A). This preference disappeared in the ambiguity condition ( $r a t e=0.51$, Wilcoxon test, $\mathrm{n}=39$, $\mathrm{p}=0.6$ ), resulting in a significant difference between the two conditions (Wilcoxon test, $\mathrm{n}=38, \mathrm{p}=8 \mathrm{e}-$ 5; mixed logit model, ambiguity dummy regressor: beta $=-1.12$, $\mathrm{z}$-value $=-7.1, \mathrm{p}=8.4 \mathrm{e}-13$, d.f. $=1444$ ). This shift was linked to a negative correlation between choosing the highest probability and the level of ambiguity ( $\mathrm{rho}=-0.25, \mathrm{p}=0.0005$, d.f. $=187$; mixed logit model, ambiguity weighted regression: beta $=-3.2$, s.e. $=0.6, \mathrm{z}$-value $=-5.0, \mathrm{p}=5-\mathrm{e}$, d.f. $=1440$; Figure $2 \mathrm{~B}$ ). Overall, participants shifted their preference from the greatest probability to the greatest reward in the ambiguity condition.

To determine whether the effect was due to changes in the probability or reward weight, we conducted logit models using the differences in probability and reward between options. The best-fit model indicated that, in no-ambiguity condition, probability and reward were considered (Logit mixed model: Probability regressor, beta $=12.8$, s.e. $=1.5$, $\mathrm{z}$-value $=8.5, \mathrm{p}<2 \mathrm{e} 16$; Reward regressor, beta $=6.2$, s.e. $=1.2, \mathrm{z}$-value $=5, \mathrm{p}=4.7 \mathrm{e}-7)$. However, ambiguity only impacted the weight of probability and not reward (Probability-ambiguity interaction, beta $=-15.5$, s.e. $=2.1, \mathrm{z}$-value $=-7.1, \mathrm{p}=1 \mathrm{e}-12$; Rewardambiguity interaction, beta $=-2.9$, s.e. $=1.5$, $\mathrm{z}$-value $=-1.8, \mathrm{p}=0.06$ ). Figure $2 \mathrm{D}$ provides further detail using Logit hierarchical Bayesian estimation, with the posterior distribution of beta probabilities having a mean of 6.7 and $95 \%$ high-density interval (HDI) of [5.7 - 7.7] ( $\mathrm{p}_{\mathrm{MCMC}}<0.001, \mathrm{p}_{\mathrm{MCMC}}$ is a pvalue derived by comparing the posterior distributions of the estimated parameters sampled via Markov Chain Monte Carlo). Similarly, the posterior distribution of beta rewards had a mean of 1.2 (HDI of $[0.8-1.7], \mathrm{p}_{\mathrm{MCMC}}<0.001$ ). The posterior probability of beta Probability-Ambiguity interaction had a mean of -3.9 and HDI of [-4.6 -3.1] ( $\left.\mathrm{p}_{\mathrm{MCMC}}<0.001\right)$. Meanwhile, the posterior beta Reward-Ambiguity interaction had a mean of -0.16 and $95 \%$ HDI of $[-0.30 .07]$ ( p $_{M C M C}=0.18$ ). In summary, the shift in behavior during ambiguous decisions appears to be associated with a selective decrease in probability weighting.

\section{Cognitive Modeling of Behavior}


Next, we investigated the potential cognitive processes underlying the observed behavioral effects. In the ambiguous condition, we anticipated that participants would allocate a portion of the concealed area $P_{a}$ to the observable probability $\left(P_{o b j}\right)$. This allocation can be characterized by two parameters, $\tau_{i}$ and $\tau_{b} . \tau_{i}$ represents the portion of $P_{a}$ assigned to $P_{o b j}$ and $\tau_{b}$ represents the laterality bias in this allocation (refer to Figure 1B). The probability that participants consider when making a decision, $P_{a l l}$, is determined by the following equations:

$$
\begin{aligned}
& P_{a l l_{l}}=\left(P_{o b j_{l}}+P_{a s_{l}}\right) /\left(P_{o b j_{l}}+P_{a s_{l}}+P_{o b j_{r}}+P_{a s_{r}}\right) \\
& P_{a s_{l}}=P_{a} * \tau_{b} * \tau_{i} \\
& P_{a s_{r}}=P_{a} *\left(1-\tau_{b}\right) * \tau_{i}
\end{aligned}
$$

Subindices 'l' and 'r' represent the left and right options, respectively. A value close to zero for $\tau_{i}$ indicates a process in which participants do not assign unknown probabilities to objective (known) probabilities, ignoring the impact of the concealed area in the decision-making process. In other words, the uncertainty introduced by the concealed area is not considered in the decision-making process.

In contrast, when $\tau_{i}$ is greater, the hidden area is taken into account during the decision-making process being assigned to objective probabilities. In the absence of bias between options, this results in a decrease in the difference between the options, increasing the uncertainty of the decision-making process. Values of $\tau_{i}$ greater than one indicate situations where participants make decisions as if they perceive more uncertainty than what is objectively generated by the hidden area (i.e., overestimation of the uncertainty). On the other hand, values of $\tau_{i}$ less than one reflect situations where participants make decisions as if they perceive less uncertainty than what is objectively generated by the concealed area (i.e., underestimation of the uncertainty).

To test whether individuals perform such computations, we fitted several cognitive computational models based on prospect theory (28). We used a hierarchical Bayesian approach $(28,29)$, and model fits were evaluated using both Deviance Information Criterion (DIC) and the leave-one-out crossvalidation information criterion (LOOIC, see Figure 2A). The models that incorporated $\tau_{i}$ and excluded $\tau_{b}$ parameters showed the best data fit (see Figure 2A). The posterior distribution of the parameter $\tau_{i}$ was greater than 1, suggesting that participants overestimated the level of ambiguity $\left(\tau_{i}\right.$ mean $=1.8 ; \mathrm{HDI}=[1.2-2.4]$; $\mathrm{p}_{\mathrm{MCMC}}=0.001$; Figure $2 \mathrm{~B}$ and Supplementary Table 1$) . \mathrm{A}$ correlation was observed between the $\tau_{i}$ value of each participant and the behavioral shift between ambiguity and no-ambiguity conditions $(\mathrm{rho}=-0.76, \mathrm{p}=3 \mathrm{e}-7, \mathrm{df}=36$; Linear regression correct for all the other model parameters, beta $=-0.24$, s.e. $=0.02$, $\mathrm{t}$-value $=-9.7, \mathrm{p}=4 \mathrm{e}-11, \mathrm{df}=32$; Figure $2 \mathrm{E}$ ), demonstrating that this parameter reflects the behavioral change between conditions. 
The posterior distribution of the parameter $\tau_{b}$ did not indicate a deviation from 0.5 , suggesting there was no laterality bias in the ambiguity probability assignment $\left(\tau_{i}+\tau_{b}\right.$ Model; $\tau_{b}$ mean $=0.5$; HDI $=\left[\begin{array}{ll}0.46 & 0.54\end{array}\right] ; \mathrm{p}_{\mathrm{MCMC}}=0.75 ;$ Figure $\left.2 \mathrm{~B}\right)$. We also conducted a controlled analysis that included a parameter ( $W_{r}$, see method) to account for potential biases toward options with greater rewards during ambiguity. The results showed no significant improvement, and the $\mathrm{Wr}$ parameter was no different from zero, indicating that there was no evidence of ambiguity biasing decisions toward options with greater rewards $\left(W_{r}\right.$ mean $=0.5 ; \mathrm{HDI}=\left[\begin{array}{ll}-0.05 & 1.3\end{array}\right] ; \mathrm{p}_{\mathrm{MCMC}}=0.08$; Figure 2B $)$.

We used the parameter readout from the $\tau_{i}$ model to simulate data using various generative $\tau_{i}$ and the same options that the experimental participants faced. Our findings showed that the generative $\tau_{i}$ can be recovered (see Supplementary Figure 2) and that higher $\tau_{i}$ parameters lead to higher payoffs (the $5 \%$ highest performance simulations out of $150000, \tau_{i}$ mode $=5.9$, median $=7.8, \mathrm{HDI}=[2.4$ $14.8])$.

\section{Cognitive Computation Robustness}

Finally, to verify the robustness of our proposed cognitive computation, we conducted a similar analysis on an independent sample of 20 participants. For this, the task was modified in several ways: the actual division of the mask was not revealed, the options were presented as two independent lotteries (while the underlying mechanism and instructions remained unchanged), and the participants made 150 decisions. The purpose was to investigate potential biases in ambiguity assignments. The results largely replicated the findings from the original sample (see Supplementary Figure 1). In the ambiguity condition, the participants shifted their decisions toward the option with the highest reward (Rate differences, Wilcoxon test, $\mathrm{n}=20, \mathrm{p}=0.001$ ), and the ambiguity degree was correlated with the decision shift (rho $=-0.58, \mathrm{p}=9 \mathrm{e}-6, \mathrm{df}=48$; mixed logit model, ambiguity weighted regression: beta $=-3.3$, s.e. $=0.5$, $\mathrm{z}$-value $=-6.4, \mathrm{p}=1 \mathrm{e}-10, \mathrm{df}=2991)$. The $\tau_{i}$ model outperformed other models, and individuals' $\tau_{i}$ values correlated with the decision shift ( rho $\left.=-0.55, \mathrm{df}=18, \mathrm{p}=0.01\right)$. No evidence was found for laterality (mean $\tau_{b}=0.51$, HDI $=\left[\begin{array}{ll}0.43 & 0.59\end{array}\right]$, $\mathrm{p}_{\mathrm{MCMC}}=0.6$ ) or reward (mean $\left.\mathrm{Wr}=0.11, \mathrm{HDI}=[-0.20 .4], \mathrm{p}_{\mathrm{MCMC}}=0.5\right)$ biases. Unlike in the first sample, the posterior distribution of the $\tau_{i}$ parameter was no different from one, indicating that in these experimental conditions, the participants did not overestimate the uncertainty induced by the ambiguity condition (mean $\tau_{i}=1.1$, $\left.\mathrm{HDI}=[0.71 .5], \mathrm{p}_{\mathrm{MCMC}}=0.6\right)$. 


\section{A}

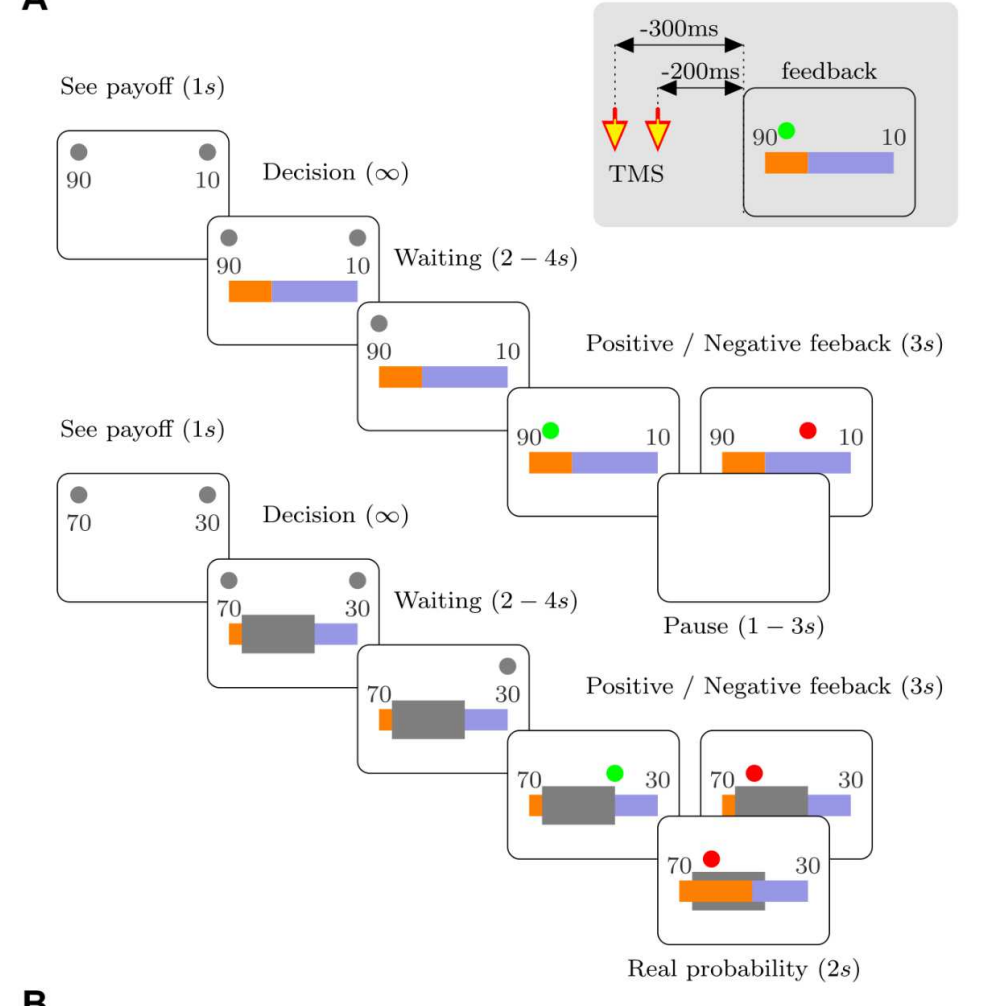

B

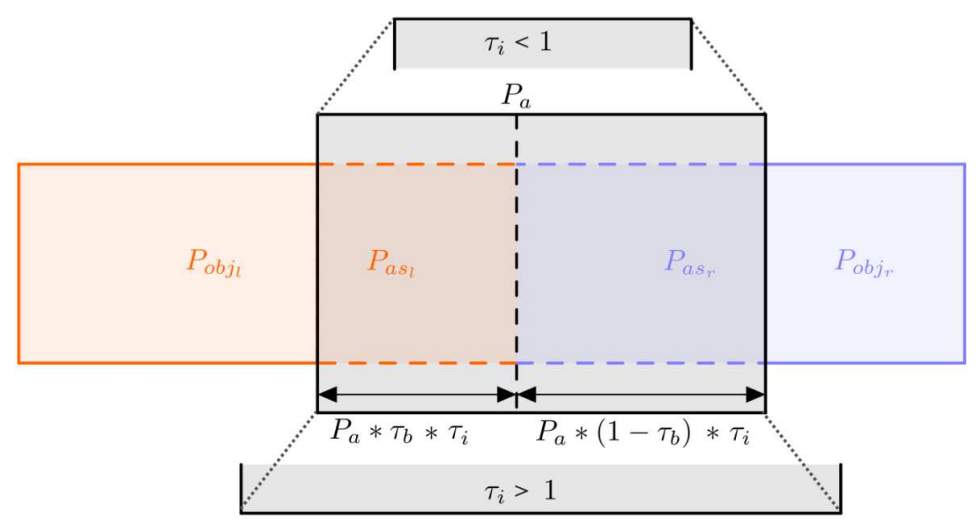

Figure 1. A. Probability Decision-Making Task. Participants were asked to decide between two options (left or right option). Each option had an associated reward indicated by a number. After a decision was made with a variable waiting time, feedback was provided. A green circle indicates that the participant won; whereas a red circle signals that he/she did not. In the ambiguity condition (bottom panel), a gray mask partially hides the color-bars extension in the division. During the TMSEEG session, a double TMS pulse is delivered -300 and $-200 \mathrm{~ms}$ before feedback presentation, as represented in the gray rectangle over the superior right corner. B. Schematic representation of the 
objective probabilities $\left(P_{o b j}\right)$ and assigned probabilities $\left(P_{a s}\right)$, and the relationship among the ambiguity probability $\left(P_{a}\right)$ and the model parameters, $\tau_{i}$ and $\tau_{b}$ (see the Results and Materials and Method sections for details).

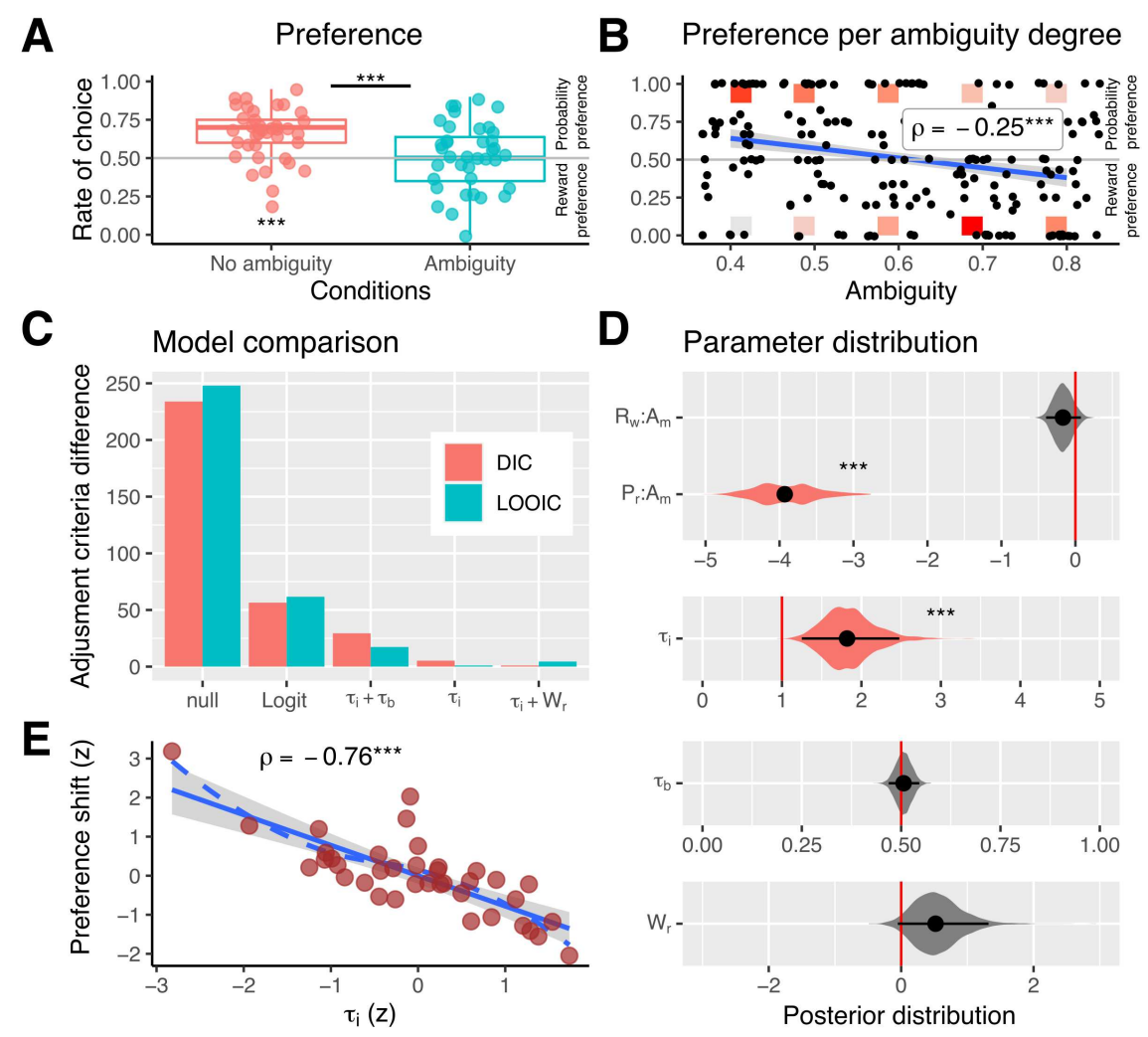

Figure 2. Behavioral Results. A. Rate of choice where individuals preferred the highest probability per conditions. B. Rate of choice where subjects preferred the highest probability per degree of ambiguity. Black dots represent the rate per individual. Color rectangles indicate the number of individual decisions; red represents the maximum; and light gray represents the minimum account. The blue line represents the linear regression, and the gray area is the standard error. $\mathbf{C}$ Model fitting comparison using DIC, red, and LOOIC, green. D Posterior distribution of the key parameters for each model. Black dots represent the mean of the distribution, and black lines the $95 \%$ high-density intervals. The colored areas represent the complete posterior distribution. E Correlation between decision shift (difference between the rate of choices that the subject prefers the highest probability between condition, ambiguity less no-ambiguity conditions), and $\tau_{i}$ parameters. Red dots represent 
each subject. The solid blue line represents the linear regression, the dotted blue line the LOESS regression, and the gray area represents the standard error. $*$ indicates $\mathrm{p}<0.05, * * \mathrm{p}<0.01,{ }^{* * *} \mathrm{p}<0.001$. See also Supplementary Figure 1 and Supplementary Table 1.

\section{fMRI}

\section{Value-related activity during decision-making}

We aimed to identify the brain regions involved in assigning probability in ambiguous situations. We used fMRI to measure brain activity during the decision phase and modeled the BOLD signal with various regressors (fMRI-Model 1: $R w, P_{\text {all }}, P_{a}$, reaction time, and ambiguity condition, see Methods for more details). Results showed that the reward magnitude of the selected option $(R w)$ increased activity in regions such as the ventral striatum, while degree of ambiguity $\left(P_{a}\right)$ correlated mainly with bilateral IPS and posterior parietal cortex (PPC). Then, we investigated brain areas correlated with the probability of the chosen option during ambiguous decisions. For this, we first explored the contrast between ambiguity and no-ambiguity conditions in the correlation with $P_{\text {all }}$ (fMRI-Model 1 , using $P_{\text {all }}$ regressors orthogonalized to $P_{a}$, and using subjects' $\tau_{i}$, see Method). This analysis revealed a unique cluster in the right IPS that correlated with the probability of the chosen option during ambiguity.

Then, we took advantage of our cognitive computational model to uncover the specific computational role of parietal areas. As $\tau_{i}$ reflects how the subject incorporates the uncertainty derived from the concealed area in the decision-making process, we used fixed $\tau_{i}$ to calculate $P_{\text {all }}$ as if subjects underestimate uncertainty $\left(\tau_{i}=0\right)$ compared with objective uncertainty $\left(\tau_{i}=1\right)$ in the decisionmaking processing. Thus, we analyzed brain activities using an fMRI model that orthogonalized these two activities $P_{\text {all }\left(\tau_{i}=0\right)}$ and $P_{\text {all }\left(\tau_{i}=1\right)}$ (fMRI-Model 2, see Methods). The results showed that the right parietal areas, including the IPS and the PCC, were uniquely correlated with $P_{\text {all }\left(\tau_{i}=0\right)}$, suggesting that these regions are involved in a computation that underestimates the uncertainty of the options. In contrast, left parietal regions correlated with both $P_{\text {all }\left(\tau_{i}=0\right)}$ and $P_{\text {all }\left(\tau_{i}=1\right)}$, and $P_{\text {all }\left(\tau_{i}=1\right)}$ also correlated with other brain areas, including the MCC and the striatum (see Supplementary Table 2 ). These findings indicate that the parietal regions play a crucial role in processing ambiguity and adjusting the level of uncertainty during decision-making. Specifically, the right parietal regions tend to compute options as more certain than they actually are.

\section{Feedback-related activity}

Using the same approach, we investigated brain activity related to feedback processing. We employed fMRI model with multiple regressors to distinguish activity related to the probability prediction error (unsigned prediction error, $\mathrm{uPE}-P_{\text {all }}$ ) and the reward prediction error (uPE- $R w$, see Methods). Results showed that winning (positive feedback) elicited activity in several brain regions, with a peak in the 
bilateral ventral striatum. For ambiguity and no-ambiguity decisions, uPE- $P_{\text {all }}$ showed correlated activity in the MCC with no significant difference between the conditions. No significant modulation was observed for uPE-Rw.

As the parietal cortex is implicated in neural computations regarding decision-making uncertainty, it should additionally influence the formation of outcome expectations and prediction errors. Hence, we would expect to observe increased connectivity between this region and areas related to outcome monitoring during feedback. Thus, we conducted a connectivity analysis (psychophysiological interaction, PPI, analysis) and tested increases in contextual connectivity of IPS (seed in joint activity for $P_{\text {all }}[\mathrm{A}>\mathrm{nA}]$ and $\left.P_{\text {all }\left(\tau_{i}=0\right)}\right)$ during ambiguity (see Methods). We found that this region increased connectivity with several outcome-monitoring areas, including the MCC and the striatum (Figure 3B).

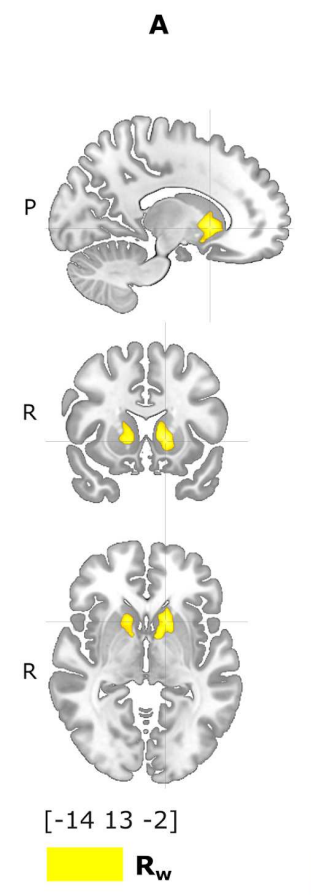

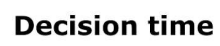
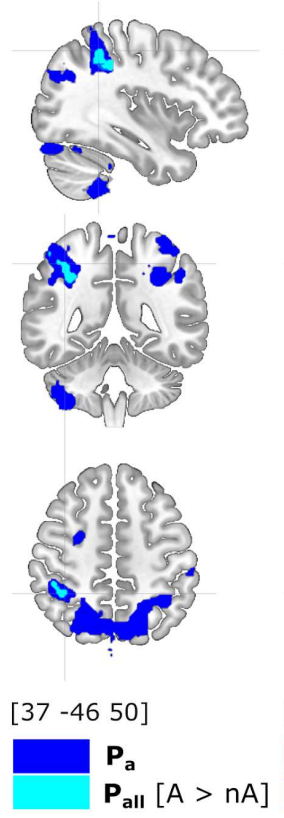
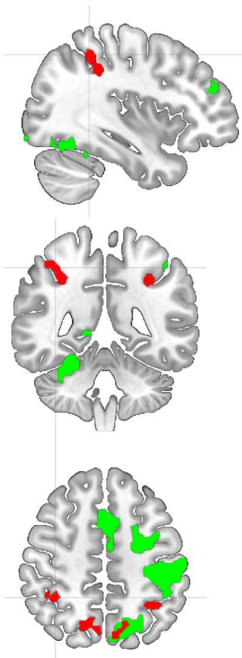

$\left[\begin{array}{lll}37 & -46 & 50\end{array}\right]$

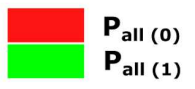

B

\section{Feedback time}
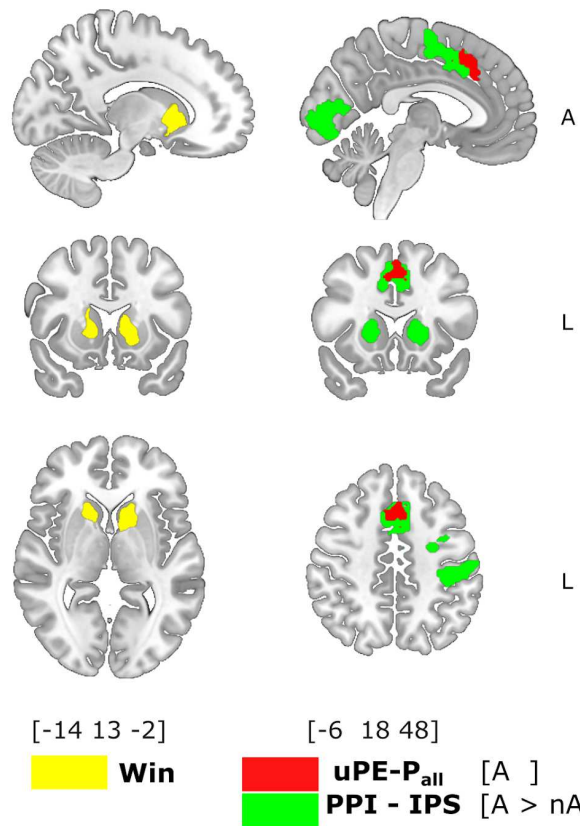

$\left[\begin{array}{lll}-6 & 18 & 48\end{array}\right]$

UPE-P all $[A]$

PPI - IPS $[A>n A]$

FIGURE 3. Brain activity during decision-making and feedback. A. Brain activity during decision-making. The reward magnitude ( $R_{w}$, yellow) of the chosen option is related to the activity in the ventral striatum (Cluster Threshold Detection (CTD) $Z=3.1$, cluster corrected $p$-value $<3 \mathrm{e}-8$, for visualization threshold $Z=4)$. Degree of ambiguity $\left(P_{a}\right.$, blue) correlates with the IPS, the PCC (cluster corrected $\mathrm{p}$-value $<1 \mathrm{e}-10)$ among other areas. The probability assigned during ambiguity correlated with the right IPS (light blue, $P_{\text {all }}[A>n A]$ : contrast $P_{\text {all }}$ during ambiguity $>P_{\text {all }}$ during 
no-ambiguity condition, corrected p-value $=0.0002$ ). Underestimating uncertainty (red, $P_{\text {all }(0)}$ calculated with tau $\mathrm{i}=0$ ) correlated bilaterally with the IPS (corrected $\mathrm{p}$-value $=0.0003$ ) and the PCC (corrected p-value $<1$ e-10). Objective uncertainty (green, $P_{\text {all }(I)}$ calculated with tau $\mathrm{i}=1$ ) correlated with the IPS, the PCC, the somatosensory area in the left hemisphere, and the SMA (all corrected p-value $<1$ e-10). B. Brain activity during feedback. The fact of winning (Win, yellow) correlated with ventral striatum activity (CTD Z $=3.1$, cluster corrected $p$-value $<1 \mathrm{e}-5$, for visualization threshold $Z=4$ ). The probability prediction during ambiguity (uPE- $P_{\text {all }}[A]$, red) correlated with activity in the MCC $(C T D Z=3.1$, cluster corrected $\mathrm{p}$-value $<1 \mathrm{e}-5)$. Contextual brain connectivity (PPI) (IPS seed from $P_{\text {all }}[A>n A]$ contrast) showed that the ambiguity condition generates an increase in the correlation between the IPS and several brain regions, including the MCC and the ventral striatum. See also Supplementary Table 2.

\section{TMS-EEG}

\section{Parietal inhibition increased the assignment of ambiguous probability.}

To investigate the causal role of the parietal cortex in ambiguous probability assignments, we conducted a TMS-EEG experiment while participants performed the same task as in the fMRI experiment. Expecting a similar behavioral effect, we targeted two regions in the parietal cortex that showed correlation with $P_{a}$ and $P_{\text {all }\left(\tau_{i}=0\right)}$, but not with $P_{\text {all }\left(\tau_{i}=1\right)}$ (as shown in Figure 4 A). These regions were the dorsal posterior parietal cortex (PPC, MNI: $[14,-64,56])$ and the right intraparietal sulcus (IPS, MNI: [46, -44, 57]). Vertex stimulation was used as a control condition. Since we found contextual connectivity between parietal and frontal regions during feedback, we designed an online TMS stimulation consisting of a doublet of pulses (separated by $100 \mathrm{~ms}$, hence covering a time window of $\sim 100-200 \mathrm{~ms}$ ) delivered trial-by-trial following the decision-making process (200 ms before the feedback onset). The rationale behind those choices was to disrupt the outcome expectancy derived from ambiguity computation, which is required to encode the prediction error during feedback. Additionally, using this protocol, we avoided interfering in the decision time in which the TMS pulse could cause motor and attentional biases, but still be able to find behavioral effects in the accumulative effect of TMS stimulation (see methods for detail of the block design).

Following the above consideration, we first aimed to examine the intra-block cumulative effect of trial-by-trial TMS on behavior. To do this, we analyzed the behavioral effects in the last 20 trials of each 40-trial block of the same TMS stimulation (see Methods). Given that the targeted areas are associated with the underestimation of the uncertainty in the decision processing (revealing by the $P_{a l l}\left(\tau_{i}=0\right)$ correlation), we hypothesized that inhibiting these areas would increase the behavioral effects of ambiguity. Consequently, both vertex and parietal TMS stimulation showed significant decision shifts between the no-ambiguity and ambiguity conditions (rate differences Vertex TMS = 0.11 , Wilcoxon test $\mathrm{p}=0.02$; Parietal TMS $=-0.2, \mathrm{p}=4 \mathrm{e}-5$ ), with parietal TMS stimulation having a greater effect (Wilcoxon test, $\mathrm{p}=0.01$ ). Parietal stimulation also increased the correlation between ambiguity degree and decision shift (linear mixed model, ambiguity and TMS interaction, beta = - 
0.7225 , s.e. $=0.3, \mathrm{z}$-value $=-2.2, \mathrm{p}=0.02, \mathrm{df}=2737$ ). There was no evidence of a difference between IPS and PPC stimulation (linear mixed model, PPC-IPS difference, beta $=0.6$, s.e. $=0.43$, z-value $=$ $1.4, \mathrm{p}=0.14, \mathrm{df}=2722$ ).

The logit model shows that parietal TMS stimulation increases the impact of the ambiguity condition on probability weighting without affecting reward weighting. The interaction between probability, ambiguity, and TMS was significant (beta $=-4.86$, s.e. $=2.3$, $\mathrm{z}$-value $=-2.1, \mathrm{p}=0.03$, $\mathrm{df}=2788$ ); meanwhile, the interaction between reward, ambiguity, and TMS was not significant (beta $=-0.2$, s.e. $=2.2$, z-value $=0.08, \mathrm{p}=0.9$ ). Logit hierarchical Bayesian estimation showed the same effects (posterior distribution of beta for probability, ambiguity, and TMS interaction: mean=-3.3, HDI $=[-$ $7.4-0.1], \mathrm{p}_{\mathrm{MCMC}}=0.027$, and posterior distribution of beta for reward, ambiguity, and TMS interaction: mean $\left.=0.3, \mathrm{HDI}=[-3.14 .2], \mathrm{p}_{\mathrm{MCMC}}=0.4\right)$.

\section{Cognitive computational modeling for TMS stimulation}

Additionally, we investigated the impact of TMS stimulation on the parameters of cognitive models. Our results showed that the $\tau_{i}$ model performed better than the other models and that TMS stimulation increased the $\tau_{i}$ parameter (posterior distribution of interaction $\tau_{i}$ and TMS, mean $=0.66, \mathrm{HDI}=[0.07$ $0.88], \mathrm{p}_{\mathrm{MCMC}}=0.008$, see Figure 4 ), and furthermore, that none of the other model parameters were affected by TMS stimulation (see Supplementary Table 3 and below). We also examined the interaction between parameters that reflect lateral bias to assess a potential attention bias generated by right parietal inhibition (30). None of these showed significant TMS modulation $\left(\beta_{o}\right.$ of softmax and TMS interaction mean $=-0.04, \mathrm{HDI}=[-0.20 .1], \mathrm{p}_{\mathrm{MCMC}}=0.6 ; \tau_{b}$ and TMS interaction mean $=0.17$, $\left.\mathrm{HDI}=\left[\begin{array}{ll}-0.03 & 0.12\end{array}\right], \mathrm{p}_{\mathrm{MCMC}}=0.17\right)$. Finally, we did not find evidence for modulation in reward estimation due to TMS stimulation ( $\alpha$ parameter of prospect theory and TMS interaction mean $=1.6$, HDI [-3.8 8.4], $\mathrm{p}_{\mathrm{MCMC}}=0.5 ; W_{r}$ and TMS interaction mean -0.1, HDI $=\left[\begin{array}{l}-0.5 \\ 0.2]\end{array}\right.$, $\left.\mathrm{p}_{\mathrm{MCMC}}=0.6\right)$. These findings suggest that the disruption of parietal activity specifically influences the assignment of ambiguous probabilities during decision-making processing without affecting other computations. 
A Target for TMS stimulation

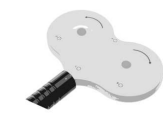

Vertex

PPC $14-6456$

IPS $\quad 46-4457$
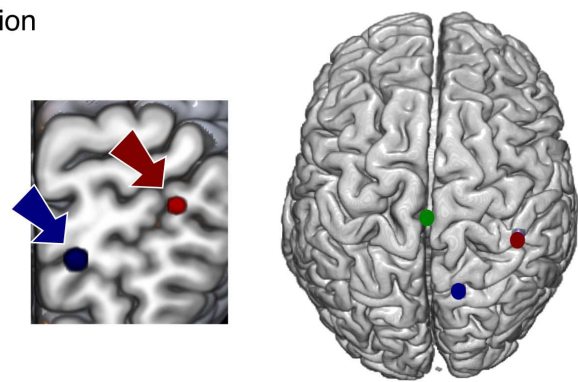

B TMS effect over preferences

C TMS effect over model parameters
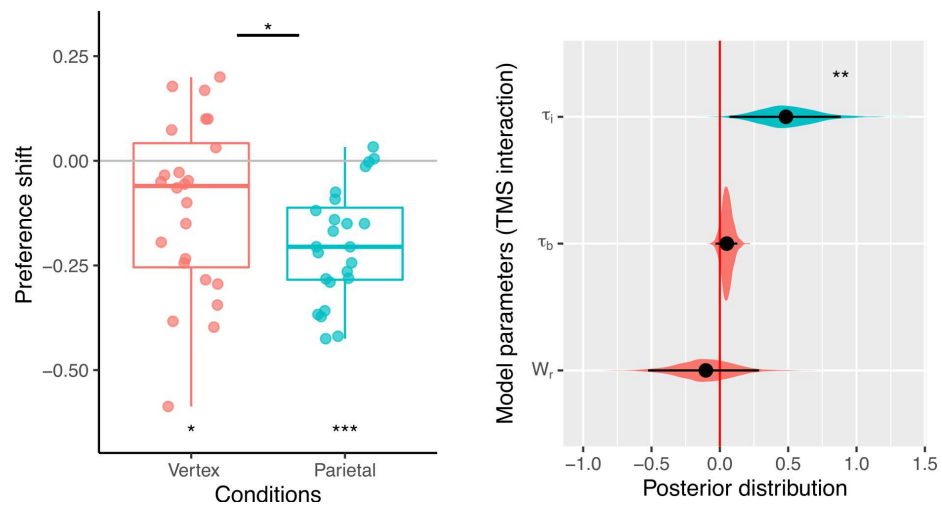

FIGURE 4. Behavioral result of interleaved EEG-TMS experiments. A. Target areas for TMS stimulation (right PPC $\mathrm{x}=14, \mathrm{y}=-64, \mathrm{z}=56$; right IPS $\mathrm{x}=46, \mathrm{y}=-44, \mathrm{z}=57$, and Scalp Vertex). B. Decision shift (difference between the rate of choices that the subject prefers with the highest probability between conditions, no-ambiguity less ambiguity) comparison between Vertex and Parietal TMS stimulation. C. Posterior distribution effect of TMS stimulation on key parameters for cognitive models. Black dots represent the mean of the distribution, and black lines the $95 \%$ highdensity intervals. The colored areas represent the complete posterior distribution. 


\section{Prediction error related activity}

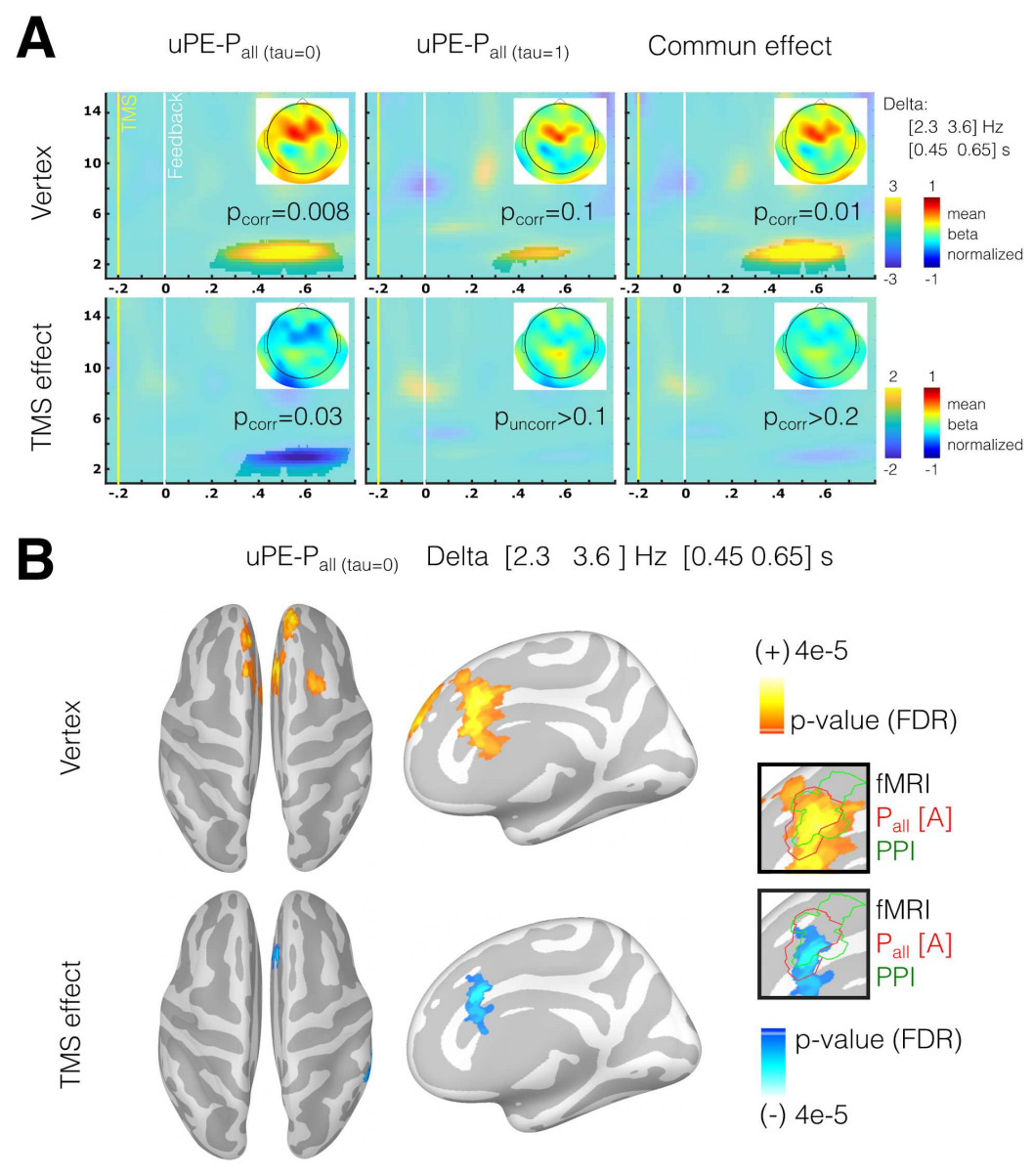

Figure 5. Oscillatory brain activity in frontal electrodes associated with unsigned prediction error during feedback. A. Time-frequency chart in frontal electrodes for the correlation between oscillatory power and unsigned prediction error given by $\tau_{i}=0$ model $\left(u P E-P_{\text {all }\left(\tau_{i}=0\right)}\right), \tau_{i}=$ $1\left(u P E-P_{a l l}\left(\tau_{i}=1\right)\right)$ model, and de join effect of two models for both Vertex TMS stimulation and the difference between vertex TMS and parietal TMS stimulation (TMS effect). The highlighted areas indicate time-frequency epochs showing significant modulation (without time-frequency a priori, cluster-based permutation test, CTD: $p<0.05$ Wilcoxon test). Scalp topographies show oscillatory activity in the delta range. B. Source estimation for delta activity correlated with unsigned prediction error given by $\tau_{i}=0$ model ( $\left.u P E-P_{a l l\left(\tau_{i}=0\right)}\right)$ for Vertex and TMS effect. Sources that survive multiple comparison corrections are shown (FDR $q<0.05$ ). The highlighted areas (green and red lines in the inserts) represent the coincident areas for EEG source estimation and BOLD activity for the fMRI experiment. All source results are shown in a high-resolution mesh only for visualization purposes. 


\section{Parietal inhibition interrupts the prediction error signal related to ambiguous probability.}

Finally, we assessed the impact of parietal inhibition on the EEG oscillatory activity during feedback. TMS stimulation showed that subjects acted as if their decisions were more uncertain (underestimation of the uncertainty). Hence, we specifically examined the signal linked to uncertainty using a model with varying $\tau_{i}$, similar to the fMRI model 2 (see Methods). We anticipated that the prediction error signal of the probability calculated with $\tau_{i}=0$, representing a lower degree of uncertainty in decision-making, would be particularly affected by parietal TMS stimulation. For this, we explored frontal electrodes where oscillatory activity related to prediction error has been described in prior work $(21-23,31)$.

The results showed that after feedback, an oscillatory activity in the delta range with a peak frequency

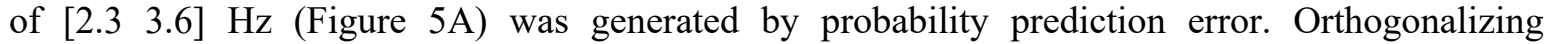
$u P E-P_{a l l\left(\tau_{i}=0\right)}$ to $u P E-P_{\operatorname{all}\left(\tau_{i}=1\right)}$ regressors, results showed that the delta activity reflected a distinct activity related to the ambiguity decision process, associated with decisions made underestimating uncertainty (cluster [1.5 4] Hz, [0.2 0.75] seconds post feedback, as determined by a cluster-based permutation test in frontal electrodes, CTD $p<0.05$, corrected $p=0.008$ ). As expected, parietal TMS stimulation disrupted this signal, resulting in a negative effect in the delta range (cluster [ 1.84$] \mathrm{Hz},\left[\begin{array}{ll}0.3 & 0.78\end{array}\right]$ seconds post-feedback, cluster-based permutation test in frontal electrodes, CTD $p<0.05$, corrected $p=0.03$ ). Source analysis revealed that the modulation caused by parietal TMS stimulation involved a similar area as found in the fMRI experiment in the MCC (as shown in the inserts in Figure 5B). Overall, the EEG results suggest that disrupting parietal activity before feedback impacts the oscillatory activity in the MCC evoked by the prediction error, suggesting a feedback processing computed as if the uncertainty of the options were overestimated. Thus, the parietal cortex plays a causal role in ambiguity computation, and parietal-to-frontal interaction is necessary for signaling the outcome predictions made through this ambiguity computation.

\section{DISCUSSION}

The results of this study provide evidence for a causal role of the parietal cortex in decision-making under ambiguous conditions. Using consecutive analyses and sequentially informed fMRI and EEGTMS experiments, we investigated the cognitive processes involved in decision-making in ambiguous situations and tested the causal involvement of the parietal cortex. We found that the subjects incorporated the uncertainty from the ambiguous information by shifting their preferences. This behavior can be explained by a model that evaluates how individuals incorporate environmental uncertainty into their decisions. Neurobiological analyses show that bilateral parietal activity was linked to objective uncertainty, while right parietal activity was linked to a process that led to incorporating less uncertainty during decision-making. Indeed, such a process is specifically affected by the interference of the right parietal activity evoked by time-locked TMS perturbation. Inhibition of the right parietal cortex showed that individuals behave as they perceive more uncertainty in the decision processing, decreasing the frontal oscillatory activity related to prediction error after making a decision under ambiguity. 
Beyond the known role of the parietal region i perceptual decision-making (32), increasing evidence has related parietal activity to value during decision-making under conditions of uncertainty $(1,33)$. Non-human primate studies have shown that parietal regions, such as the intraparietal sulcus (IPS), link the probability of obtaining a reward with a specific action (e.g., the direction of the saccade (34)). Neurons of the dorsal parietal region have also shown activity for a combination of reward magnitude and probability (1). Moreover, some parietal neurons are specifically modulated by the expected utility of the options (35). Following this notion, research comparing human decisionmaking models has shown a selectivity of the parietal cortex in encoding expected utility (i.e., the weight of the reward given by the subjective probability as expressed in Prospect Theory (33)). In this context, our results show differential modulations of the parietal cortex associated with the chosen option probability depending on the objective and perceived uncertainty. Research on nonhuman primates suggests that parietal neurons are highly attuned to the level of uncertainty in a perceptual decision-making task (13). In addition, these findings have highlighted the critical role played by the parietal cortex in encoding information about the potential reduction of uncertainty that could result from a particular behavior or decision (13). Taken together, these studies suggest that the parietal cortex is able to gauge the predictability of reward by distinguishing between what is already known and what remains uncertain.

Our experimental approach found a causal role of the parietal cortex in the computation of ambiguous options, even though human studies have shown conflicting results on its role in decision-making under uncertainty. In accordance with our results, patients with posterior parietal lesions have been found to be less able to adjust their decision-making strategies based on the probability of winning compared to patients with frontal lesions, suggesting a potential causal role of the posterior parietal cortex in decision-making (36). On the other hand, parietal activity has been related to a surprise signal with a general effect of cognitive reallocation, for example, slowing reaction time, but not with a value process (14). In addition, other studies have reported a correlation between parietal activity and value processing only in specific demanding circumstances, for example, time pressure (37). Parietal activity has also been correlated with the belief update, reducing the degree of ambiguity rather than value update (12). In this context, our results support a causal engagement of the parietal cortex in value processing under uncertain situations, as parietal interference by TMS affected a particular computation related to ambiguity management. Additionally, parietal suppression reduced the signal related to prediction error associated with ambiguous probabilities in the medial prefrontal cortex. Since there is no learning in our experimental task, we cannot rule out whether this activity is just a surprise signal related to expectation violation or has a role in value updating and learning. Nevertheless, in both cases, it is necessary to build an expectation that incorporates environmental ambiguity. The results of our study suggest that the parietal cortex is causally involved in such a process. Further research is necessary to better understand the exact nature and mechanisms of the involvement of partial computation in the learning process.

Our results suggest a specific computation that may occur in the parietal cortex when faced with ambiguity although other research may reveal alternative interpretations. Under ambiguity, the parietal activation can also be interpreted as sensing the necessity to reduce uncertainty throughout learning, valuing, and categorizing. Thus, interrupting parietal activity could impair categorization 
processing, generating more straightforward decision rules. Recent research in mice has shown a causal role for the parietal cortex in new but not well-learned sensory stimuli categorization (38). The parietal cortex takes part in learning and categorization processes before new stimuli have been incorporated into existing categories (38). The correlation between parietal cortex activity and the degree of ambiguity in the decision revealed by our findings might be associated with a process to reduce the uncertainty that an ambiguous stimulus evokes. Thus, parietal activity may play a role in using previous knowledge and experience in categorical choices $(19,38)$. Thus, the effect of parietal TMS perturbation could be interpreted as using a simpler heuristic with less categorization processing. For instance, the subject simply chooses the option based on the associated reward. Heuristics are crucial in complex situations because they are simplified decision rules that help individuals deal with problems requiring high cognitive investment $(39,40)$. Compared with younger adults, older adults show different parietal activity when faced with a decision under uncertain probability and different heuristic decision-making (41). Comparative studies indicate that nonhuman primates, as do adult humans, show ambiguity aversion, revealing that this situation entails a high cognitive cost $(2,3)$. Interestingly, most primate species use simple heuristics to face ambiguous decisions, and only great apes consider the ambiguity of the information in the decision process (39). In accordance, researchers in developmental neuroscience have shown that children do not show ambiguity aversion (42) as adults and adolescents do (43). A recent study shows that the IPS implements complex heuristics in sequential decision-making tasks (44). In light of our current results, the interference of the parietal cortex can be interpreted here as preventing the use of more complex computation for managing ambiguity.

Considering the preceding evidence, the correlation between parietal activity and the degree of uncertainty can reflect a high cognitive demand. Indeed, previous findings have shown that regardless of the sensory properties of the stimuli, parietal activity has been largely related to numeric magnitude $(45,46)$. The IPS has been associated with several numerical and spatial operations in humans, including arithmetic calculations and spatial rotation (47). Thus, the IPS appears to be a highly sensitive region for the processing and manipulation of magnitudes across various dimensions, including abstract numbers, space, and time $(47,48)$. In our results, the parietal interference operates on a specific parameter in the computational model related to the incorporation of the uncertainty of ambiguous information. Although such an operation might involve a high cognitive demand, the parietal activity was independent of other proxies of difficulty, such as reaction time. We also found no evidence that parietal stimulation generated a laterality bias in choice. The preceding is important since the right IPS has been related to spatial attention (30). According to fMRI research, parietal activity during decision-making under uncertainty is not influenced by general attentional load (49). In this context, parietal activity seems to be better understood as a specific computation of uncertainty rather than as a general cognitive load.

During outcome monitoring, we report frontal oscillatory activity related to expectation violation based on sensing less uncertainty in the decision under ambiguity. Prefrontal oscillatory activity has a widely studied role in cognitive control and working memory $(25,50-52)$. Extensive research has shown that prefrontal delta and theta activity correlates with prediction errors $(18,21-23,53-56)$. Prior evidence showed that parietal and frontal areas sustain effective connectivity during and after a decision, and such activity biases follow decision-making $(19,20)$. Research using the EEG technique has demonstrated that frontal oscillatory activity correlates with the uncertainty and unexpectedness 
of an event $(21,22)$ and that it is associated with future exploration strategies $(21)$. According to prior research, the source of this oscillatory activity is in the $\operatorname{MCC}(23,57)$. It has been hypothesized that MCC contains multiple circuits participating in sensing diverse and relevant internal and environmental variables, such as information about reward, punishment, and uncertainty (58). Moreover, this area is part of a network involving basal ganglia that present activity related to outcome uncertainty (59). Specifically, this network has been proposed as regulating behavior aiming to obtain uncertainty-resolving information (59). Studies have found that when stimuli have information that was important to resolving uncertainty, it affects visual search behavior $(60,61)$. Interestingly, both MCC and the parietal cortex have been linked to such behavior $(13,61)$. Thus, the IPS - MCC connectivity generated by the ambiguous information could be interpreted as a mechanism to contrast and update the uncertainty of the chosen event. Notably, intolerance to uncertainty has largely been related to anxiety, a pervasive symptom that presents in a broad spectrum of psychiatry and neurological diseases (62). Accordingly, EEG frontal oscillatory activity reflecting MCC activity is moderated by anxiety and predicts adaptive behavioral adjustments under uncertainty (63).

In summary, we took advantage of the sequential use of fMRI and TMS-EES studies to localize and interfere with model-derived signals related to the use of ambiguous probabilities to provide causation. Our results demonstrate a causal implication of the parietal cortex in managing ambiguity during decision-making and assigning uncertainty during decision-making processing. Additionally, we tested whether the localized perturbation in the parietal cortex spreads through the cortex and alters neural processing in remote areas. Specifically, we demonstrated a decrease in the signal related to violation expectation in the MCC once participants evaluated the outcome of their decisions. As a result, the evidence provided here contributes to generating deep insight into the cognitive and neural mechanisms underlying decision-making in situations of ambiguity. Notably, difficulties dealing with uncertainty or ambiguity commonly result in anxiety (62). Hence, the mechanism we identified here could become a potential target for further studies in several neuropsychiatric symptoms that have been associated with the perception and the computation of uncertainty, such as those present in Autism Spectrum (64) and Obsessive-compulsive Disorders (65).

\section{MATERIALS AND METHODS}

\section{Participants}

Seventy-four healthy participants between the ages of 18 and 45 participated in the experimental protocol approved by the Ethics Committee of the Universidad del Desarrollo, Chile. Thirty-nine participants took part in the fMRI session, 24 participated in the EEG-TMS session ( 9 of them also participated in the fMRI session), and 20 in a behavioral replication sample. All had normal or corrected to normal vision, no color vision impairment, no history of neurological disease, and no current psychiatric diagnosis or psychotropic prescriptions. All participants gave informed consent. Experiments were conducted in the Social Neuroscience and Neuromodulation Laboratory at the Centro de Investigación en Complejidad Social (neuroCICS) at the Universidad del Desarrollo, the Unidad de Imágenes Cuantitativas Avanzadas (UNICA) at the Clínica Alemana de Santiago, and Grenoble Institut Neurosciences at the Univ. Grenoble Alpes, Inserm, U1216. 


\section{Task}

All participants completed the probabilistic decision-making (PDM) task (66) in which they had to choose between two probabilistic options with rewards. Each option was represented by a bar color (on each side of the screen) and associated with a probability of being selected, represented by the length of a colored bar placed in the center of the screen, and a reward, represented by a number placed above each colored bar. These numbers represent real monetary incentives (see below). The options had random, complementary probabilities and rewards, with the option having the highest visible bar (highest probability) having the lowest reward and vice versa. After the participant had made a selection ( $\sim 2$ to 6 seconds), the rewarded option was indicated with either a green circle if participants chose the rewarded option or with a red circle otherwise. Feedback presentation (red or green circle) lasted for 3 seconds. If the participant chose the rewarded option, they received the associated reward, otherwise, they received no money. Participants completed this task under two conditions: no-ambiguity and ambiguity. In the former condition, participants saw the full extension of the color bar, with complete information related to the probability distribution of possible outcomes (i.e., risk or first-order uncertainty). In the latter condition, a gray mask partially hid part of the extension of both bars. The size of this mask could vary from $40 \%$ to $80 \%$. In these cases, participants had incomplete information about the probability distribution of possible outcomes (i.e., ambiguity or second-order uncertainty). The task was programmed and presented using Presentation Software (Neurobehavioral Systems TM).

In the fMRI experimental session, participants completed 40 trials: 20 for the no-ambiguity condition and 20 for the ambiguity condition in 5-trial blocks. In the TMS-EEG experimental session, participants completed 240 trials in 10-trial blocks per condition (no-ambiguity and ambiguity). Each participant completed 6 runs of TMS stimulations, consisting of 2 runs of 40 trials with TMS interference on the PPC (MNI x=14, $y=-64, z=56)$, two runs of 40 trials with TMS interference at the IPS (MNI x=46, $y=-44, z=57$ ), and two runs of 40 trials with TMS interference at the vertex, as an active control condition. The order of these 6 runs was randomly selected for each participant. Stimulation was applied 200 and 300 ms before the Feedback epoch with a double inhibitory pulse separated by $100 \mathrm{~ms}$. The TMS target regions were calculated based on group analyses of the fMRI session. For the behavioral replication sample, the options were presented as two independent lotteries. During the ambiguity condition, the areas behind the mask were not revealed in order to explore for possible bias. Each participant completed 150 trials in 5-trial blocks per condition (noambiguity and ambiguity), with a break every 50 trials.

\section{Statistical analysis of the behavior}

The participants' answers were analyzed with a computational cognitive approach. All computational cognitive models were fitted using prospect theory, which assumes that the following equation defines the expected subjective value $U_{l}$ of an option (indicates left option) that individuals use to make a decision. 


$$
U_{l}=v\left(x_{l}\right) \pi\left(P_{a l l_{l}}\right)-v\left(x_{r}\right) \pi\left(P_{a l l_{r}}\right)
$$

In equation 4, subindices ' 1 ' and ' $r$ ' represent the left and right options, respectively. $v($.$) represents$ the value function, $x_{l}$ and $x_{r}$ denote the potential outcome of each option associated with the left or right option, respectively. We used the following equation,

$$
v(x)=x^{\alpha}
$$

where $\alpha$ determines the concavity of the value function. $P_{a l l_{l}}$ and $P_{a l l_{r}}$ are the probabilities of a gain, whereas $\pi($.$) are the subjective decision weights assigned to these probabilities. To accommodate$ for the existence of unknown probabilities (i.e., for ambiguity condition), the probability $P_{\text {all }}$ by which the outcome $\mathrm{x}$ occurs is defined by the equations $1,2,3$, and 6 (see results).

$$
\pi\left(P_{\text {all }}\right)=\frac{P_{\text {all }}^{\gamma}}{\left(P_{\text {all }}^{\gamma}+\left(1-P_{\text {all }}\right)^{\gamma}\right)^{1 / \gamma}}
$$

The extent by which the ambiguity area $P_{a}$ is assigned to each option is modulated by two parameters: $\tau_{i}$ that represents the ratio of the $P_{a}$ effectively assigned, and $\tau_{b}$ that represents the ratio by which the subject biases one of the two options. The models where $\tau_{b}$ was set to 0.5 involving a process of unbiased (homogeneous) assignment between options (left or right). Additionally, we explored an alternative bias parameter Wr that influenced the reward estimation ( $\alpha$ parameter of Prospect Theory, equation 5) under ambiguity given by the following equation:

$$
v\left(x_{l}\right)= \begin{cases}x_{l}^{(\alpha+W r)} & \text { if } x_{l} \geq x_{r} \\ x_{l}(\alpha) & \text { if } x_{l}<x_{r}\end{cases}
$$

The probability of choosing the left option for a given subjective value is computed using a logistic choice rule wherein $\beta_{1}$ is an inverse temperature parameter representing the degree of stochasticity in the choice process and $\beta_{0}$ is a bias parameter.

$$
\theta\left(U_{l}\right)=\frac{1}{1+e^{-\beta_{l}\left(U_{l}-\beta_{0}\right)}}
$$

All parameters were estimated using a Hierarchical Bayesian approach that uses the aggregated information from the entire population sample to inform and constrain the parameter estimates for each individual. The hierarchical structure contains two levels of random variation: the trial (i) and participant (s) levels. At the trial level, choices were modeled following a Bernoulli process: 


$$
y(s, i) \sim \operatorname{bern}\left(\theta\left(U_{l}-U_{r}\right)\right)
$$

At the participant level, the model parameters were constrained by group-level hyper-parameters.

The parameters $\tau_{b}$ were restricted to be between 0 and 1 using a Beta distribution.

$$
\tau_{b(s)} \sim \operatorname{beta}\left(\mu_{\tau_{b}} \kappa_{\tau_{b}},\left(1-\mu_{\tau_{b}}\right) \kappa_{\tau_{b}}\right)
$$

Where $\mu_{\tau}$ represents the mean and $\kappa_{\tau}$ represents the dispersion of the beta distribution.

The parameters $\beta$ and $\mathrm{Wr}$ at the participant level were parameterized using normal distributions. The $\tau_{i}, \alpha$ and $\gamma$ parameters at the participant level were also parameterized using normal distributions and restricted to positive values.

$$
\begin{aligned}
& \alpha_{(s)} \sim \operatorname{normal}\left(\mu_{\alpha}, \sigma_{\alpha}\right) \\
& \beta_{(s)} \sim \operatorname{normal}\left(\mu_{\beta}, \sigma_{\beta}\right) \\
& \gamma_{(s)} \sim \operatorname{normal}\left(\mu_{\gamma}, \sigma_{\gamma}\right)
\end{aligned}
$$

We assumed flat distributions for each parameter at the highest level of the hierarchy (hyperparameters).

$$
\begin{array}{r}
\mu_{\left(\alpha, \tau_{i}, \gamma\right)} \sim \operatorname{unif}(0.01,100) \\
\mu_{(W r, \beta)} \sim \operatorname{normal}(0,100) \\
\sigma \sim \operatorname{unif}(0.001,100) \\
\mu_{\tau_{b}} \sim \operatorname{beta}(1,1) \\
\kappa_{\tau_{b}} \sim \text { unif }(0.001,100)
\end{array}
$$


The posterior inference of the parameters in the hierarchical Bayesian models was performed via the Gibbs sampler using the Markov Chain Monte Carlo (MCMC) technique, which was implemented in JAGS using R software. A minimum of 10,000 samples were drawn from an initial burn-in sequence. Subsequently, a total of 10,000 new samples were drawn using three chains, each of which was derived based on a different random number generator engine using different seeds. We increased the length of the burn-in sequence if the chains did not meet the criteria for convergence, as outlined below. We applied a thinning of 10 to this sample, resulting in a final set of 3,000 samples for each parameter. This thinning was used to avoid autocorrelation among the final samples for the parameters of interest. We conducted Gelman-Rubin tests for each parameter to confirm the convergence of the chains. All latent variables in our models had a Gelman-Rubin statistic near 1, which suggests that all three chains converged to the target posterior distribution.

Additionally, the behavior was also analyzed using mixed-effect logistic regression, assuming no specific ambiguity computation.

$$
\text { Left } \sim P_{o b j_{l}}+R w_{l}+P_{a}+P_{o b j_{l}}: P_{a}+P_{o b j_{l}}: P_{a}
$$

The full interaction logistic model was tested but presented higher AICs, indicating worse adjustments.

\section{Anatomical Data}

All participants underwent a 3D anatomical MPRAGE T1-weighted and T2-weighted Magnetic Resonance Imaging scan on a 3T Siemens Skyra (Siemens AG, Erlangen, Germany) no more than 3 months before the TMS-EEG sessions or together with the fMRI sessions. The anatomical volume consisted of 160 sagittal slices of an isotropic voxel $(1 \mathrm{x} 1 \mathrm{x} 1 \mathrm{~mm})$, covering the whole brain. The scalp and cortical surfaces were extracted from the T1-weighted/T2-weighted corrected anatomical MRI using a pipeline available from the Human Connectome Project. Thus, a surface triangulation was obtained for each envelope (67). The individual high-resolution cortical surfaces $(\sim 300,000$ vertices per cortical surface) were down sampled to $\sim 8,000$ vertices. Additionally, a five-layer segmentation based on T1-weighted and T2-weighted was carried out using the algorithm implemented by the SimNIBS tool and SMP12. The cortical mesh and five-layer segmentation served as image supports for the EEG source estimation. (See below.)

\section{Functional MRI Data}

For the functional images, volumes of the entire weighted echo-planner $\mathrm{T} 2 *$ brain were acquired while the experimental task was executed ( $3 \times 3 \times 3 \mathrm{~mm}$ voxels). Participant volumes were coregistered to 2-mm standard imaging using the nonlinear algorithm implemented in FSL. The BOLD signal was analyzed using different models, including motion correction parameters (MC). During decisionmaking periods, we fitted two models as follows.

\section{fMRI Model 1:}


fMRI Model 2:

$$
\mathrm{BOLD} \sim \mathrm{P}_{\mathrm{all}[\mathrm{A}]}+\mathrm{P}_{\mathrm{all}[\mathrm{nA}]}+\mathrm{P}_{\mathrm{a}}+\mathrm{Rw}+\mathrm{RT}+\mathrm{Am}+\mathrm{MC}
$$

$$
\mathrm{BOLD} \sim \mathrm{P}_{\mathrm{all}}\left(\tau_{i=0)}+\mathrm{P}_{\mathrm{all}} \tau_{i=1)}+\mathrm{P}_{\mathrm{a}}+\mathrm{Rw}+\mathrm{RT}+\mathrm{Am}+\mathrm{MC}\right.
$$

Am is a dummy regressor capturing the "state" or baseline activity that the participants had in the ambiguity condition, and the RT is a reaction-time regressor as a proxy of difficulty. For model $1, \mathrm{P}_{\text {all }}$ for both conditions (Ambiguity [A] and No- ambiguity [nA]) was orthogonalized to $P_{a}$ in order to obtain unique activity related to probability computation independent of the degree of ambiguity. Additionally, in fMRI-Model $2 \mathrm{P}_{\text {all }} \tau_{i=0)}$ and $\left.\mathrm{P}_{\text {all }} \tau_{i}=1\right)$ were orthogonalized with each other in order to obtain their independent contribution to the signal during the ambiguity condition.

For the BOLD signal during outcome evaluation (feedback), we used the following regressors of interest: Win (a dummy regressor indicating that the chosen option was rewarded), $R w$ (the amount of the obtained reward), uPE- $P_{a l l[A]}$ (the unsigned prediction error of the fact to win or not to win given by the $P_{\text {all }}$ of the chosen option in Ambiguity conditions), uPE- $P_{\text {all }[n A]}$ (the unsigned prediction error of the fact to win or not to win given by the $P_{\text {all }}$ of the chosen option in no-ambiguity conditions), uPE- $R w$ (the unsigned prediction error of the amount of the obtained reward). All regressors were convolved using a double gamma function.

\section{EEG Recordings}

We used TMS-compatible EEG equipment (BrainAmp 64 DC, BrainProducts, http://www. brainproducts.com/). EEG was continuously acquired from 64 channels (plus an acquisition reference (FCz) and a ground). TMS-compatible sintered $\mathrm{Ag} / \mathrm{AgCl}$-pin electrodes were used. The signal was band-pass filtered at DC to $1000 \mathrm{~Hz}$ and digitized at a sampling rate of $5000 \mathrm{~Hz}$. Skin/electrode impedance was maintained below $5 \mathrm{k} \Omega$. Electrode impedances were re-tested during pauses to ensure stable values throughout the experiment. The positions of the EEG electrodes were estimated using the neuronavigation system used for the TMS.

\section{EEG-TMS Protocol}

TMS was applied during task performance and during EEG recordings. Participants were instructed to maintain central fixation and minimize eye blinks and other movements during the recording blocks. Double biphasic TMS pulses were delivered over the right IPS (TMS ips, $_{\text {, MI }}[46,-44,57]$ ), the right PPC $\left(\mathrm{TMS}_{\mathrm{ppc}}\right.$, MNI $\left.[14,-64,56]\right)$, and the Vertex $\left(\mathrm{TMS}_{\text {vertex }}\right.$, MNI $[0,-29,77]$; see Results, Figures 3 and 4A) using a $70 \mathrm{~mm}$ figure-of-eight TMS coil connected to Mag and More Stimulator. A neuronavigation system was used to identify individual stimulation points (individual structural MR scans, native space) in the nearest gray matter areas to the no-linear inverse co-registration of the individual anatomy (FSL algorithm, default parameters). TMS coil positioning and orientation with regards to brain $\mathrm{x}, \mathrm{y}$, and $\mathrm{z}$ axes ( $\mathrm{yaw}$, pitch, and roll) were optimized so that the electric field impacted perpendicular to the target region, maximizing the induced current strength $(68,69)$. This approach results for all subjects with approximately an angulation in a horizontal plane (yaw) with regards to the interhemispheric fissure of $45^{\circ}$ for the IPS and $0^{\circ}$ for the PPC and the vertex. For each trial and 
for both tasks, two consecutive single TMS pulses were delivered before the feedback presentation (300 and $-200 \mathrm{~ms}$ pre-stimulus onset) with an interpulse interval of $100 \mathrm{~ms}$ in order to interfere with target activity with a 100-200 ms window that has been used in prior work $(70,71)$ and has been demonstrated to inhibit motor potential (72). TMS intensity was fixed at $120 \%$ of the individual resting motor threshold (TMS intensity ranging from $54 \%$ to $78 \%$ of the maximum machine power and a mean of $63 \%$ ). Each TMS session included six runs. In each run, 40 two-pulse TMS bursts were delivered trial by trial, leading to 80 pulses per run over a block duration of about $11 \mathrm{~min}$. Pauses for a minimum of 5 minutes duration separated each run. Each TMS-EEG experiment thus contained a total of 480 active TMS pulses (including those delivered at the vertex). Two 5-min EEG restingstate recordings were performed before and after the six blocks. The duration of the experiment was around 180 minutes: one hour for setting the EEG electrodes at stable and adequate impedances, one and half hours of recordings, and 30 minutes for the electrode MRI localization and experiment finalization. The TMS protocol respected at all times past and current safety recommendations regarding stimulation parameters (intensity, number of pulses, and ethical requirements (73-75)).

\section{EEG Pre-processing and TMS Artifact Removal}

Preprocessing was performed in multiple steps. We first detected the slow decay component of the TMS artifact. To this end, we segmented 1-second windows containing TMS pulses, automatically detected a period starting $10 \mathrm{~ms}$ pre to $20 \mathrm{~ms}$ post to the respective TMS peak and removed this from the signal. We applied an Independent Component Analysis (ICA) to this signal using the Runica algorithm provided by the EEGLAB toolbox (https://sccn.ucsd.edu/eeglab/). Thus, we looked for a stereotype component with local bipolar distribution over the TMS site pulse. In the second step, we segmented the raw signal in the time widows of analysis ( -1.5 seconds to 2 seconds after feedback onset). Then, we removed the segment between -10 to $30 \mathrm{~ms}$ around the TMS peak and replaced it with an inverse-distance weighted interpolation $\left[\mathrm{Y}=\operatorname{sum}\left(\mathrm{X} / \mathrm{D}^{\wedge} 3\right) / \operatorname{sum}\left(1 / \mathrm{D}^{\wedge} 3\right)\right]$ plus a Gaussian noise with the standard deviation extracted to a reference period set to be 55 to $-15 \mathrm{~ms}$ before the respective first TMS peak of the double pulse and 0 of the mean. Then, we removed the TMS ICA components computed in the first step. This procedure effectively removed the direct (non-physiological) and other TMS artifacts (e.g., TMS-locked artifacts at electrodes directly in contact with the TMS coil) without introducing discontinuities, important for the later time-frequency analysis $(68,76)$. Following these steps, we down-sampled EEG data to $1000 \mathrm{~Hz}$ and used a preprocessing pipeline developed for prior work $(51,52,77-79)$. The EEG data was $0.1-45 \mathrm{~Hz}$ band-pass filtered. Artifacts were first automatically detected using a threshold of $150 \mu \mathrm{V}$ and a power spectrum greater than two std. dev. for more than $10 \%$ of the frequency spectrum $(0.5$ to $40 \mathrm{~Hz})$. Blinking was extracted from the signal by means of ICA. Remaining trials that included artifacts detected by visual inspection of the signal were eliminated. The mean of artifact-free trials was 229 out of 240, ranges: [182 240]. Finally, the signal was re-referenced offline to the average of all electrodes for the subsequent analyses.

Time-frequency (TF) distributions were obtained by means of the wavelet transform in a time window between -1.5 and $2 \mathrm{~s}$ around feedback onset. To this end, the signal $\mathrm{x}(\mathrm{t})$ was convolved with a complex Morlet's wavelet function. Wavelets were normalized, and the width of each wavelet function was chosen to be five cycles. Thus, we obtained the phase and amplitude per each temporal bin (in steps of $10 \mathrm{~ms}$ ) and frequency (from 1 to $40 \mathrm{~Hz}$ in steps of $1 \mathrm{~Hz}$ ). For all power spectrum analyses, we used 
the $\mathrm{dB}$ of power related to a baseline during the fixation phase (at the beginning of the experiments). To avoid edge artifacts, only the period between -0.25 to 0.8 s over the segmented signals was used for additional analyses.

We calculated general linear models for each subject based on single-trial wavelet transform (firstlevel analysis). We used the following regressor for this analysis: Win (dummy regressor), $R w$, uPE$P_{\text {all }\left(\tau_{i}=0\right)}$, uPE- $P_{\text {all }\left(\tau_{i}=1\right)}$, uPE- $R w, A m$ (dummy regressor), and a regressor for each TMS stimulation $\left(T M S_{i p s}, T M S_{p p c}\right)$ and the interaction between the TMS regressor with the preceding regressors. Thus, per each regressor and subject, we obtained a 3D matrix (time, frequency, electrode), which we used in the second-level analysis. For the analyses of the frontal electrodes of interest, the 3D matrix, including only the selected ones (Fz, CFz, Cz, F1, FC1, C1, F2, FC2, C2), was averaged in the electrode dimension. We explored consistent modulations in the same condition. For this, we used the Wilcoxon signed sum test, evaluating whether the mean is different from zero. All comparisons were corrected for multiple comparisons using a cluster-based permutation test (see below) (80) or false discovery rate (FDR) for a priori selection of a frequency-time window of interest in source analysis).

\section{Cluster-based Permutation test}

In order to correct for multiple comparisons in the time-frequency analysis, we carried out a permutation test (80). Here, clusters of significant areas were defined by pooling neighboring sites that showed the same effect $(\mathrm{p}<0.05$ in the statistical test carried out in sites of either the timefrequency chart or the sources, e.g., Wilcoxon test). The cluster-level statistics were computed as the sum of the statistics of all sites within the corresponding cluster. We evaluated the cluster-level significance under the permutation distribution of the cluster that had the largest cluster-level statistics. The permutation distribution was obtained by randomly permuting the original data. Specifically, for each subject, we carried out null models, wherein the same structure of the original model was preserved, but the regressor tested was permuted. After each permutation, the original statistical test was computed (e.g., Wilcoxon), and the cluster-level statistics of the largest resulting cluster were used for the permutation distribution. After 5000 permutations, the cluster-level significance of each observed cluster was estimated as the proportion of elements of the permutation distribution greater than the cluster-level statistics of the corresponding cluster.

\section{EEG Source Estimation}

The neural current density time series at each brain location were estimated by applying a minimum norm estimate inverse solution LORETA algorithm with unconstrained dipole orientations in singletrial signal per condition and subject, implemented in Brainstorm. A tessellated cortical mesh for individual anatomy was used as a brain model to estimate the current source distribution. We defined approximately $3 \times 8000$ sources constrained to the segmented cortical surface ( 3 orthogonal sources at each spatial location). We computed a five-layer continuous Galerkin finite element conductivity model (FEM), as implemented in DUneuro software (78), and the physical forward model. To estimate cortical activity at the cortical sources, the recorded raw EEG time series at the electrodes were multiplied by the inverse operator to yield the estimated source current at the cortical surface as 
a function of time. Since this is a linear transformation, it does not modify the frequencies of the underlying sources. It is, therefore, possible to undertake time-frequency analysis on the source space directly. In this source space, we first reduced the dipole of each vertex to one, selecting the component with the greater variance using the PCA algorithm. We then computed frequency decomposition using the Wavelets transform. To minimize the possibility of erroneous results, we only present source estimations if there are statistically significant differences at both the electrode and source levels (i.e., differences that survive multiple comparison corrections).

\section{Data Availability}

The complete minimal data set underlying the results used in our study are available in the public repository of the OSF website https://osf.io/zd3g7 under the name of our manuscript -A causal role for the parietal cortex in ambiguity computations in humans (doi: 10.17605/OSF.IO/G2EA3). The additional toolbox and codes used in the analysis are available on our lab git site at https://github.com/neurocics/ and the OSF website https://osf.io/zd3g7.

\section{ACKNOWLEDGMENTS}

We want to thank Leonie Kausel and Anne Bliss for proofreading the manuscript. This work was supported by Agencia Nacional de Investigación y Desarrollo de Chile (ANID), FONDECYT (1181295 and 1211227 to P.B.), PAI (PAI77190047 to P.S-I), ECOS/CONICYT (C12S03), FONDEQUIP EQM150076.

\section{AUTHOR CONTRIBUTIONS}

Conceptualization, P.B., G.V-O., A.V-C. and R.P.; Methodology, P.B., R.P., J.B., and G.V-O.; Formal Analysis, P.B., G.V-O. and R.P., Investigation G.V-O., A.F-V., M.M-M., and P.B.; Resources F.Z., C.S., J.L-V. and X.S.; Writing P.B., P.S-I., G.V-O., R.P., and A.V-C.; Project Administration P.B., G.V-O. and A.F-V.; Funding Acquisition J.B., P.B.

\section{DECLARATION OF INTEREST}

The authors declare no competing interests.

\section{REFERENCES}

1. P. N. Tobler, E. U. Weber, Neuroeconomics (Second Edition). Part Ii Neural Psychological Found Econ Preferences, 149-172 (2014).

2. B. Y. Hayden, S. R. Heilbronner, M. L. Platt, Ambiguity Aversion in Rhesus Macaques. Front Neurosciswitz. 4, 166 (2010).

3. A. G. Rosati, B. Hare, Chimpanzees and bonobos distinguish between risk and ambiguity. Biol Letters. 7, 15-18 (2011).

4. C. Camerer, M. Weber, Recent developments in modeling preferences: Uncertainty and ambiguity. $J$ Risk Uncertainty. 5, 325-370 (1992).

5. D. Ellsberg, Risk, Ambiguity, and the Savage Axioms. Q J Econ. 75, 643 (1961).

6. K. Juechems, C. Summerfield, Where Does Value Come From? Trends Cogn Sci. 23, 836-850 (2019).

7. A. Lopez-Persem, J. Bastin, M. Petton, R. Abitbol, K. Lehongre, C. Adam, V. Navarro, S. Rheims, P. Kahane, P. Domenech, M. Pessiglione, Four core properties of the human brain valuation system demonstrated in intracranial signals. Nat Neurosci. 23, 664-675 (2020). 
8. C. Padoa-Schioppa, K. E. Conen, Orbitofrontal Cortex: A Neural Circuit for Economic Decisions. Neuron. 96, 736-754 (2017).

9. D. C. Farrar, A. Z. Mian, A. E. Budson, M. B. Moss, R. J. Killiany, Functional brain networks involved in decision-making under certain and uncertain conditions. Neuroradiology. 60, 61-69 (2018).

10. S. A. Huettel, A. W. Song, G. McCarthy, Decisions under Uncertainty: Probabilistic Context Influences Activation of Prefrontal and Parietal Cortices. J Neurosci. 25, 3304-3311 (2005).

11. E. Payzan-LeNestour, S. Dunne, P. Bossaerts, J. P. O'Doherty, The Neural Representation of Unexpected Uncertainty during Value-Based Decision Making. Neuron. 79, 191-201 (2013).

12. K. Kobayashi, M. Hsu, Neural Mechanisms of Updating under Reducible and Irreducible Uncertainty. $J$ Neurosci Official J Soc Neurosci. 37, 6972-6982 (2017).

13. M. Horan, N. Daddaoua, J. Gottlieb, Parietal neurons encode information sampling based on decision uncertainty. Nat Neurosci. 22, 1327-1335 (2019).

14. J. X. O'Reilly, U. Schüffelgen, S. F. Cuell, T. E. J. Behrens, R. B. Mars, M. F. S. Rushworth, Dissociable effects of surprise and model update in parietal and anterior cingulate cortex. Proc National Acad Sci. 110, E3660-E3669 (2013).

15. A. Rangel, C. Camerer, P. R. Montague, A framework for studying the neurobiology of value-based decision making. Nat Rev Neurosci. 9, 545-556 (2008).

16. C. Padoa-Schioppa, Orbitofrontal Cortex and the Computation of Economic Value. Ann Ny Acad Sci. 1121, 232-253 (2007).

17. J. W. Kable, P. W. Glimcher, The Neurobiology of Decision: Consensus and Controversy. Neuron. 63, 733-745 (2009).

18. M. C. M. Gueguen, A. Lopez-Persem, P. Billeke, J.-P. Lachaux, S. Rheims, P. Kahane, L. Minotti, O.

David, M. Pessiglione, J. Bastin, Anatomical dissociation of intracerebral signals for reward and punishment prediction errors in humans. Nat Commun. 12, 3344 (2021).

19. R. Akaishi, K. Umeda, A. Nagase, K. Sakai, Autonomous Mechanism of Internal Choice Estimate Underlies Decision Inertia. Neuron. 81, 195-206 (2014).

20. R. Polanía, M. Moisa, A. Opitz, M. Grueschow, C. C. Ruff, The precision of value-based choices depends causally on fronto-parietal phase coupling. Nat Commun. 6, 8090 (2015).

21. J. F. Cavanagh, C. M. Figueroa, M. X. Cohen, M. J. Frank, Frontal Theta Reflects Uncertainty and Unexpectedness during Exploration and Exploitation. Cereb Cortex. 22, 2575-2586 (2012).

22. P. Billeke, F. Zamorano, D. Cosmelli, F. Aboitiz, Oscillatory Brain Activity Correlates with Risk Perception and Predicts Social Decisions. Cereb Cortex. 23, 2872-2883 (2013).

23. P. Billeke, F. Zamorano, T. López, C. Rodriguez, D. Cosmelli, F. Aboitiz, Someone has to give in: theta oscillations correlate with adaptive behavior in social bargaining. Soc Cogn Affect Neur. 9, 2041-2048 (2014).

24. F. Zamorano, L. Kausel, C. Albornoz, C. Lavin, A. Figueroa-Vargas, X. Stecher, D. Aragón-Caqueo, X. Carrasco, F. Aboitiz, P. Billeke, Lateral Prefrontal Theta Oscillations Reflect Proactive Cognitive Control Impairment in Males With Attention Deficit Hyperactivity Disorder. Frontiers Syst Neurosci. 14, 37 (2020). 25. J. F. Cavanagh, M. J. Frank, Frontal theta as a mechanism for cognitive control. Trends Cogn Sci. 18, 414-421 (2014).

26. J. D. Murray, J. Jaramillo, X.-J. Wang, Working Memory and Decision-Making in a Frontoparietal Circuit Model. J Neurosci. 37, 12167-12186 (2017).

27. M. Hsu, M. Bhatt, R. Adolphs, D. Tranel, C. F. Camerer, Neural Systems Responding to Degrees of 
Uncertainty in Human Decision-Making. Science. 310, 1680-1683 (2005).

28. M. G. Edelson, R. Polania, C. C. Ruff, E. Fehr, T. A. Hare, Computational and neurobiological foundations of leadership decisions. Sci New York N Y. 361, eaat0036 (2018).

29. C. A. Hill, S. Suzuki, R. Polania, M. Moisa, J. P. O’Doherty, C. C. Ruff, A causal account of the brain network computations underlying strategic social behavior. Nat Neurosci. 20, 1142-1149 (2017).

30. C. R. Gillebert, D. Mantini, V. Thijs, S. Sunaert, P. Dupont, R. Vandenberghe, Lesion evidence for the critical role of the intraparietal sulcus in spatial attention. Brain. 134, 1694-1709 (2011).

31. P. Verbeke, K. Ergo, E. D. Loof, T. Verguts, Learning to Synchronize: Midfrontal Theta Dynamics during Rule Switching. J Neurosci. 41, 1516-1528 (2020).

32. J. I. Gold, H. R. Heekeren, Neuroeconomics (Second Edition). Part Iv Neural Mech Choice, 355-372 (2014).

33. T. B. Williams, C. J. Burke, S. Nebe, K. Preuschoff, E. Fehr, P. N. Tobler, Testing models at the neural level reveals how the brain computes subjective value. Proc National Acad Sci. 118, e2106237118 (2021).

34. L. P. Sugrue, G. S. Corrado, W. T. Newsome, Matching Behavior and the Representation of Value in the Parietal Cortex. Science. 304, 1782-1787 (2004).

35. M. C. Dorris, P. W. Glimcher, Activity in Posterior Parietal Cortex Is Correlated with the Relative Subjective Desirability of Action. Neuron. 44, 365-378 (2004).

36. B. Studer, F. Manes, G. Humphreys, T. W. Robbins, L. Clark, Risk-Sensitive Decision-Making in Patients with Posterior Parietal and Ventromedial Prefrontal Cortex Injury. Cereb Cortex New York Ny. 25, $1-$ 9 (2015).

37. G. Jocham, P. M. Furlong, I. L. Kröger, M. C. Kahn, L. T. Hunt, T. E. J. Behrens, Dissociable contributions of ventromedial prefrontal and posterior parietal cortex to value-guided choice. Neuroimage. 100, 498-506 (2014).

38. L. Zhong, Y. Zhang, C. A. Duan, J. Deng, J. Pan, N. Xu, Causal contributions of parietal cortex to perceptual decision-making during stimulus categorization. Nat Neurosci. 22, 963-973 (2019).

39. A. Romain, M.-H. Broihanne, A. D. Marco, B. Ngoubangoye, J. Call, N. Rebout, V. Dufour, Non-human primates use combined rules when deciding under ambiguity. Philosophical Transactions Royal Soc B. 376, 20190672 (2021).

40. E. Brandstätter, G. Gigerenzer, R. Hertwig, The Priority Heuristic: Making Choices Without Trade-Offs. Psychol Rev. 113, 409-432 (2006).

41. S. M. H. Hosseini, M. Rostami, Y. Yomogida, M. Takahashi, T. Tsukiura, R. Kawashima, Aging and decision making under uncertainty: Behavioral and neural evidence for the preservation of decision making in the absence of learning in old age. Neuroimage. 52, 1514-1520 (2010).

42. R. Li, E. M. Brannon, S. A. Huettel, Children do not exhibit ambiguity aversion despite intact familiarity bias. Front Psychol. 5, 1519 (2015).

43. N. E. Blankenstein, S. A. Huettel, R. Li, Resolving ambiguity: Broadening the consideration of risky decision making over adolescent development. Dev Rev. 62, 100987 (2021).

44. C. W. Korn, D. R. Bach, Heuristic and optimal policy computations in the human brain during sequential decision-making. Nat Commun. 9, 325 (2018).

45. A. Nieder, I. Diester, O. Tudusciuc, Temporal and Spatial Enumeration Processes in the Primate Parietal Cortex. Science. 313, 1431-1435 (2006).

46. A. Nieder, S. Dehaene, Representation of Number in the Brain. Annu Rev Neurosci. 32, 185-208 (2009).

47. Z. Hawes, H. M. Sokolowski, C. B. Ononye, D. Ansari, Neural Underpinnings of Numerical and Spatial 
Cognition: An fMRI Meta-Analysis of Brain Regions Associated with Symbolic Number, Arithmetic, and Mental Rotation. Neurosci Biobehav Rev. 103, 316-336 (2019).

48. H. M. Sokolowski, W. Fias, C. B. Ononye, D. Ansari, Are numbers grounded in a general magnitude processing system? A functional neuroimaging meta-analysis. Neuropsychologia. 105, 50-69 (2017).

49. T. J. Vickery, Y. V. Jiang, Inferior Parietal Lobule Supports Decision Making under Uncertainty in Humans. Cereb Cortex. 19, 916-925 (2009).

50. E. K. Miller, J. D. Cohen, An Integrative Theory of Prefrontal Cortex Function. Annu Rev Neurosci. 24, 167-202 (2001).

51. J. Larrain-Valenzuela, F. Zamorano, P. Soto-Icaza, X. Carrasco, C. Herrera, F. Daiber, F. Aboitiz, P. Billeke, Theta and Alpha Oscillation Impairments in Autistic Spectrum Disorder Reflect Working Memory Deficit. Sci Rep-uk. 7, 14328 (2017).

52. A. Figueroa-Vargas, C. Cárcamo, R. Henríquez-Ch, F. Zamorano, E. Ciampi, R. Uribe-San-Martin, M. Vásquez, F. Aboitiz, P. Billeke, Frontoparietal connectivity correlates with working memory performance in multiple sclerosis. Sci Rep-uk. 10, 9310 (2020).

53. D. M. Barch, T. S. Braver, F. W. Sabb, D. C. Noll, Anterior Cingulate and the Monitoring of Response Conflict: Evidence from an fMRI Study of Overt Verb Generation. J Cognitive Neurosci. 12, 298-309 (2000). 54. C. W. Hoy, S. C. Steiner, R. T. Knight, Single-trial modeling separates multiple overlapping prediction errors during reward processing in human EEG. Commun Biology. 4, 910 (2021).

55. S. Mei, Q. Li, X. Liu, Y. Zheng, Monetary Incentives Modulate Feedback-related Brain Activity. Sci Rep-uk. 8, 11913 (2018).

56. J. F. Cavanagh, M. J. Frank, T. J. Klein, J. J. B. Allen, Frontal theta links prediction errors to behavioral adaptation in reinforcement learning. Neuroimage. 49, 3198-209 (2009).

57. Z. Fu, A. Sajad, S. P. Errington, J. D. Schall, U. Rutishauser, Neurophysiological mechanisms of error monitoring in human and non-human primates. Nat Rev Neurosci, 1-20 (2023).

58. I. E. Monosov, Anterior cingulate is a source of valence-specific information about value and uncertainty. Nat Commun. 8, 134 (2017).

59. I. E. Monosov, How Outcome Uncertainty Mediates Attention, Learning, and Decision-Making. Trends Neurosci. 43, 795-809 (2020).

60. N. Daddaoua, M. Lopes, J. Gottlieb, Intrinsically motivated oculomotor exploration guided by uncertainty reduction and conditioned reinforcement in non-human primates. Sci Rep-uk. 6, 20202 (2016).

61. J. K. White, E. S. Bromberg-Martin, S. R. Heilbronner, K. Zhang, J. Pai, S. N. Haber, I. E. Monosov, A neural network for information seeking. Nat Commun. 10, 5168 (2019).

62. D. W. Grupe, J. B. Nitschke, Uncertainty and anticipation in anxiety: an integrated neurobiological and psychological perspective. Nat Rev Neurosci. 14, 488-501 (2013).

63. J. F. Cavanagh, A. J. Shackman, Frontal midline theta reflects anxiety and cognitive control: Metaanalytic evidence. J Physiology-paris. 109, 3-15 (2015).

64. E. Stark, J. Stacey, W. Mandy, M. L. Kringelbach, F. Happé, Autistic Cognition: Charting Routes to Anxiety. Trends Cogn Sci. 25, 571-581 (2021).

65. M. M. Vaghi, F. Luyckx, A. Sule, N. A. Fineberg, T. W. Robbins, B. D. Martino, Compulsivity Reveals a Novel Dissociation between Action and Confidence. Neuron. 96, 348-354.e4 (2017).

66. P. Billeke, T. Ossandon, M. Perrone-Bertolotti, P. Kahane, J. Bastin, K. Jerbi, J.-P. Lachaux, P. Fuentealba, Human Anterior Insula Encodes Performance Feedback and Relays Prediction Error to the Medial Prefrontal Cortex. Cereb Cortex. 30, 4011-4025 (2020). 
67. B. Fischl, FreeSurfer. Neuroimage. 62, 774-781 (2012).

68. G. Thut, D. Veniero, V. Romei, C. Miniussi, P. Schyns, J. Gross, Rhythmic TMS Causes Local

Entrainment of Natural Oscillatory Signatures. Curr Biol. 21, 1176-1185 (2011).

69. A. Valero-Cabré, J. L. Amengual, C. Stengel, A. Pascual-Leone, O. A. Coubard, Transcranial magnetic stimulation in basic and clinical neuroscience: A comprehensive review of fundamental principles and novel insights. Neurosci Biobehav Rev. 83, 381-404 (2017).

70. A. B. Chica, P. Bartolomeo, A. Valero-Cabre, Dorsal and Ventral Parietal Contributions to Spatial Orienting in the Human Brain. J Neurosci. 31, 8143-8149 (2011).

71. R. Oshio, S. Tanaka, N. Sadato, M. Sokabe, T. Hanakawa, M. Honda, Differential effect of double-pulse TMS applied to dorsal premotor cortex and precuneus during internal operation of visuospatial information. Neuroimage. 49, 1108-1115 (2010).

72. R. Chen, E. M. Wassermann, M. Caños, M. Hallett, Impaired inhibition in writer's cramp during voluntary muscle activation. Neurology. 49, 1054-1059 (1997).

73. S. Rossi, M. Hallett, P. M. Rossini, A. Pascual-Leone, T. S. of T. C. Group, Safety, ethical considerations, and application guidelines for the use of transcranial magnetic stimulation in clinical practice and research. Clin Neurophysiol. 120, 2008-2039 (2009).

74. P. M. Rossini, D. Burke, R. Chen, L. G. Cohen, Z. Daskalakis, R. D. Iorio, V. D. Lazzaro, F. Ferreri, P. B. Fitzgerald, M. S. George, M. Hallett, J. P. Lefaucheur, B. Langguth, H. Matsumoto, C. Miniussi, M. A. Nitsche, A. Pascual-Leone, W. Paulus, S. Rossi, J. C. Rothwell, H. R. Siebner, Y. Ugawa, V. Walsh, U. Ziemann, Non-invasive electrical and magnetic stimulation of the brain, spinal cord, roots and peripheral nerves: Basic principles and procedures for routine clinical and research application. An updated report from an I.F.C.N. Committee. Clin Neurophysiology Official J Int Fed Clin Neurophysiology. 126, 1071-1107 (2015).

75. S. Rossi, A. Antal, S. Bestmann, M. Bikson, C. Brewer, J. Brockmöller, L. L. Carpenter, M. Cincotta, R. Chen, J. D. Daskalakis, V. D. Lazzaro, M. D. Fox, M. S. George, D. Gilbert, V. K. Kimiskidis, G. Koch, R. J. Ilmoniemi, J. P. Lefaucheur, L. Leocani, S. H. Lisanby, C. Miniussi, F. Padberg, A. Pascual-Leone, W. Paulus, A. V. Peterchev, A. Quartarone, A. Rotenberg, J. Rothwell, P. M. Rossini, E. Santarnecchi, M. M. Shafi, H. R. Siebner, Y. Ugawa, E. M. Wassermann, A. Zangen, U. Ziemann, M. Hallett, T. basis of this article began with a C. S. from the I. W. on "Present 2020 Future of TMS: Safety, Ethical Guidelines", Siena, October 17-20, 2018, updating through April, Safety and recommendations for TMS use in healthy subjects and patient populations, with updates on training, ethical and regulatory issues: Expert Guidelines. Clin Neurophysiol. 132, 269-306 (2021).

76. P. Albouy, A. Weiss, S. Baillet, R. J. Zatorre, Selective Entrainment of Theta Oscillations in the Dorsal Stream Causally Enhances Auditory Working Memory Performance. Neuron. 94, 193-206.e5 (2017).

77. P. Soto-Icaza, L. Vargas, F. Aboitiz, P. Billeke, Beta oscillations precede joint attention and correlate with mentalization in typical development and autism. Cortex. 113, 210-228 (2019).

78. P. Billeke, A. Armijo, D. Castillo, T. López, F. Zamorano, D. Cosmelli, F. Aboitiz, Paradoxical Expectation: Oscillatory Brain Activity Reveals Social Interaction Impairment in Schizophrenia. Biol Psychiat. 78, 421-31 (2014).

79. P. Billeke, F. Zamorano, M. Chavez, D. Cosmelli, F. Aboitiz, Functional Cortical Network in Alpha Band Correlates with Social Bargaining. Plos One. 9, e109829 (2014).

80. E. Maris, R. Oostenveld, Nonparametric statistical testing of EEG- and MEG-data. J Neurosci Meth. 164, 177-190 (2007). 


\section{Supplementary Materials}

Supplementary Tables 1, 2 and 3

Supplementary Figures 1 and 2 


\section{Supplementary Table 1}

\section{fMRI behavioral Models}

MODEL: Logit

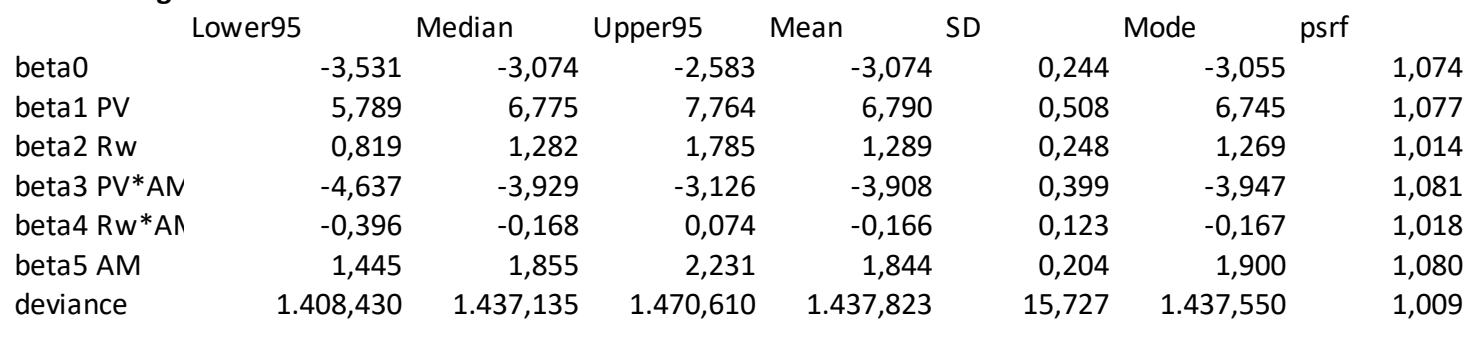

MODEL: null (Prospect theory)

\begin{tabular}{|c|c|c|c|c|c|c|c|}
\hline & Lower95 & Median & Upper95 & Mean & SD & Mode & psrf \\
\hline alpha & 1,102 & 1,602 & 2,056 & 1,610 & 0,245 & 1,614 & 1,012 \\
\hline gamma & 1,802 & 2,522 & 3,374 & 2,549 & 0,408 & 2,485 & 1,006 \\
\hline beta_0 & $-0,043$ & 0,088 & 0,215 & 0,086 & 0,068 & 0,085 & 1,003 \\
\hline peat_1 & 6,454 & 10,426 & 15,155 & 10,652 & 2,231 & 10,014 & 1,019 \\
\hline deviance & $1.599,340$ & $1.626,300$ & $1.657,740$ & $1.626,962$ & 14,966 & $1.625,050$ & 1,004 \\
\hline
\end{tabular}

MODEL: tau_i

\begin{tabular}{|c|c|c|c|c|c|c|c|}
\hline & Lower95 & Median & Upper95 & Mean & SD & Mode & psrf \\
\hline tau_i & 1,250 & 1,819 & 2,478 & 1,848 & 0,322 & 1,794 & 1,002 \\
\hline alpha & 0,699 & 1,115 & 1,678 & 1,133 & 0,252 & 1,089 & 1,002 \\
\hline gamma & 2,552 & 3,890 & 5,475 & 3,900 & 0,729 & 3,923 & 1,010 \\
\hline peta_0 & 0,012 & 0,150 & 0,282 & 0,148 & 0,068 & 0,145 & 1,006 \\
\hline eta_1 & 11,750 & 22,486 & 42,344 & 24,469 & 8,264 & 20,948 & 1,011 \\
\hline eviance & $1.330,300$ & $1.363,900$ & $1.396,900$ & $1.364,200$ & 17,092 & $1.363,400$ & 1,001 \\
\hline
\end{tabular}

\begin{tabular}{|c|c|c|c|c|c|c|c|}
\hline \multicolumn{8}{|c|}{ MODEL: tau_i + tau_b } \\
\hline & Lower95 & Median & Upper95 & Mean & SD & Mode & psrf \\
\hline tau_b & 0,468 & 0,507 & 0,547 & 0,507 & 0,021 & 0,507 & 1,001 \\
\hline tau_i & 0,989 & 1,451 & 1,971 & 1,474 & 0,259 & 1,403 & 1,002 \\
\hline alpha & 0,608 & 1,097 & 1,791 & 1,151 & 0,313 & 1,015 & 1,010 \\
\hline gamma & 2,113 & 3,661 & 5,552 & 3,765 & 0,883 & 3,475 & 1,007 \\
\hline beta_0 & $-179,972$ & 2,547 & 207,589 & 2,651 & 99,241 & 7,229 & 1,000 \\
\hline beat_1 & 10,422 & 20,533 & 55,917 & 24,283 & 12,639 & 18,495 & 1,021 \\
\hline deviance & $1.322,880$ & $1.358,205$ & $1.394,250$ & $1.358,304$ & 18,387 & $1.358,770$ & 1,000 \\
\hline
\end{tabular}

\begin{tabular}{|c|c|c|c|c|c|c|c|}
\hline \multicolumn{8}{|c|}{ MODEL: tau_i + Wr } \\
\hline & Lower95 & Median & Upper95 & Mean & SD & Mode & psrf \\
\hline tau_i & 1,247 & 1,853 & 2,614 & 1,893 & 0,348 & 1,791 & 1,006 \\
\hline alpha & 0,800 & 0,957 & 1,162 & 0,970 & 0,108 & 0,889 & 1,001 \\
\hline $\mathrm{Er}$ & $-0,122$ & 0,488 & 1,287 & 0,531 & 0,362 & 0,445 & 1,011 \\
\hline $\mathrm{Wr}$ & 0,165 & 2,539 & 4,254 & 2,425 & 1,140 & 2,850 & 1,001 \\
\hline beta_o & 0,014 & 0,163 & 0,313 & 0,163 & 0,076 & 0,174 & 1,016 \\
\hline beta_1 & 13,031 & 20,098 & 29,514 & 20,816 & 4,691 & 19,407 & 1,007 \\
\hline deviance & $1.325,420$ & $1.357,860$ & $1.392,880$ & $1.358,047$ & 17,281 & $1.357,207$ & 1,002 \\
\hline
\end{tabular}




\section{Supplementary Table 2}

\section{fMRI Decision Model 1}

Regressor: Rw

\begin{tabular}{|c|c|c|c|c|c|c|c|c|c|c|}
\hline Cluster & Voxels & $\mathbf{P}$ & $-\log 10(P)$ & Z-MAX & Z-MAX X & Z-MAX Y & Z-MAX Z & Z-COG X & Z-COG Y & Z-COG Z \\
\hline 19 & 2473 & $9,90 \mathrm{E}-25$ & 24 & 5 & 2 & 20 & 50 & $-3,6$ & 15 & 50 \\
\hline 18 & 2225 & $5,45 \mathrm{E}-23$ & 22,3 & 4,38 & -44 & -30 & 36 & $-37,8$ & $-43,6$ & \\
\hline 17 & 1012 & $3,04 \mathrm{E}-13$ & 12,5 & 4,25 & 28 & -54 & -24 & 24,4 & $-65,2$ & \\
\hline 16 & 961 & $9,12 \mathrm{E}-13$ & 12 & 5,28 & 16 & 18 & 10 & 14,1 & 6,95 & \\
\hline 15 & 949 & $1,19 \mathrm{E}-12$ & 11,9 & 5,22 & -12 & 6 & 0 & $-13,8$ & 10,2 & \\
\hline 14 & 358 & $3,40 \mathrm{E}-06$ & 5,47 & 4,29 & 50 & -70 & 2 & 51,1 & $-65,7$ & \\
\hline 13 & 326 & $9,12 \mathrm{E}-06$ & 5,04 & 4,03 & -54 & 14 & 0 & $-51,7$ & 9,56 & \\
\hline 12 & 315 & $1,29 \mathrm{E}-05$ & 4,89 & 4,31 & -42 & 54 & 12 & $-40,2$ & 50,1 & \\
\hline 11 & 304 & $1,82 \mathrm{E}-05$ & 4,74 & 4,29 & 30 & -38 & 44 & 38,6 & $-41,1$ & \\
\hline 10 & 299 & $2,15 \mathrm{E}-05$ & 4,67 & 4,23 & 36 & -2 & 52 & 32,9 & $-3,98$ & \\
\hline 9 & 211 & $4,25 \mathrm{E}-04$ & 3,37 & 4,15 & -14 & -18 & 4 & $-10,8$ & $-18,8$ & \\
\hline 8 & 207 & $4,92 \mathrm{E}-04$ & 3,31 & 4,36 & 60 & 14 & 2 & 54,5 & 11 & \\
\hline 7 & 195 & $7,63 \mathrm{E}-04$ & 3,12 & 3,97 & 12 & -70 & 56 & 16,6 & $-68,9$ & \\
\hline 6 & 179 & $1,39 \mathrm{E}-03$ & 2,86 & 4,37 & -32 & -68 & -24 & $-32,9$ & $-58,6$ & \\
\hline 5 & 168 & $2,13 \mathrm{E}-03$ & 2,67 & 4,17 & 28 & -76 & -50 & 24,5 & $-74,2$ & \\
\hline 4 & 149 & $4,52 \mathrm{E}-03$ & 2,35 & 4,18 & 42 & 48 & 16 & 40,6 & 47,4 & \\
\hline 3 & 146 & $5,10 \mathrm{E}-03$ & 2,29 & 3,91 & 20 & -100 & 6 & 16,7 & -101 & \\
\hline 2 & 127 & $1,12 \mathrm{E}-02$ & 1,95 & 4,03 & 2 & -58 & -24 & 2,84 & $-60,8$ & \\
\hline 1 & 105 & $2,94 \mathrm{E}-02$ & 1,53 & 4,35 & -30 & 22 & 2 & -30 & 21,2 & \\
\hline
\end{tabular}

$\begin{array}{rrrrr}\text { COPE- } & \text { COPE- } & \text { COPE- } & \text { COPE- } & \text { COPE- } \\ 106 & -32 & -4 & 70 & 37,3 \\ 138 & -48 & -40 & 60 & 46,6 \\ 69,3 & 28 & -76 & -18 & 30,7 \\ 53,7 & 8 & 6 & 4 & 23,2 \\ 63,2 & -6 & 2 & 10 & 26,2 \\ 49,2 & 52 & -66 & 0 & 28,9 \\ 67 & -54 & 18 & -4 & 35 \\ 55,2 & -42 & 54 & 12 & 33,6 \\ 76,2 & 48 & -38 & 62 & 41,4 \\ 87,5 & 32 & 4 & 66 & 40,2 \\ 31 & -2 & -12 & 10 & 15,8 \\ 53,1 & 60 & 14 & 2 & 29,6 \\ 82,2 & 12 & -70 & 62 & 49,9 \\ 36,8 & -32 & -68 & -22 & 23,5 \\ 30,8 & 30 & -74 & -50 & 23,8 \\ 38,9 & 42 & 52 & 12 & 29,7 \\ 57,5 & 12 & -102 & 0 & 40,2 \\ 19,7 & 4 & -66 & -38 & 15,9 \\ 31,8 & -32 & 20 & 8 & 21,5\end{array}$

\section{Regressor: Pa}

\begin{tabular}{|c|c|c|c|c|c|c|c|c|c|c|c|c|c|c|c|}
\hline $\begin{array}{c}\text { Cluster } \\
5\end{array}$ & $\begin{array}{c}\text { Voxels } \\
6009\end{array}$ & $\begin{array}{c}\mathbf{P} \\
0,00 \mathrm{E}+00\end{array}$ & $-\log 10(P)$ & $\begin{array}{c}\text { Z-MAX } \\
5,37\end{array}$ & $\underset{-16}{\text { Z-MAX X }}$ & $\begin{array}{c}\text { Z-MAX Y } \\
-64\end{array}$ & $\underset{50}{\text { Z-MAX Z }}$ & $\underset{1,35}{\text { Z-COG X }}$ & $\begin{array}{c}\text { Z-COG Y } \\
-59,5\end{array}$ & $\begin{array}{c}\text { Z-COG Z } \\
53,1\end{array}$ & $\underset{245}{\text { COPE- }}$ & $\begin{array}{c}\text { COPE- } \\
10\end{array}$ & $\begin{array}{c}\text { COPE- } \\
-68\end{array}$ & $\underset{64}{\text { COPE- }}$ & $\begin{array}{c}\text { COPE- } \\
101\end{array}$ \\
\hline 4 & 1157 & $1,81 \mathrm{E}-15$ & 14,7 & 5,07 & 12 & -92 & -22 & 23,1 & $-80,7$ & $-22,8$ & 257 & 20 & -90 & -22 & 95,4 \\
\hline 3 & 381 & $6,56 \mathrm{E}-07$ & 6,18 & 5,03 & 36 & -48 & -54 & 36 & -45 & $-50,1$ & 81,7 & 36 & -48 & -54 & 49,9 \\
\hline 2 & 159 & $1,85 \mathrm{E}-03$ & 2,73 & 4,18 & -28 & -64 & -28 & $-28,9$ & $-64,3$ & $-27,2$ & 64,8 & -26 & -74 & -22 & 47,9 \\
\hline 1 & 132 & $5,97 \mathrm{E}-03$ & 2,22 & 4,51 & 28 & -4 & 52 & 27,3 & $-4,6$ & 52,5 & 79.4 & 30 & -6 & 58 & 56,1 \\
\hline
\end{tabular}

Regressor: Pall $[\mathbf{A}>\mathbf{n A}]$

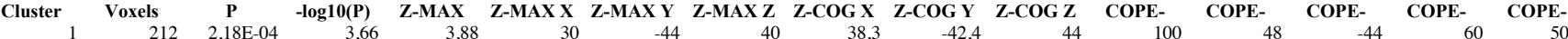

\section{fMRI Decision Model 2}

\section{Regressor: Pall(tau_i=0)}

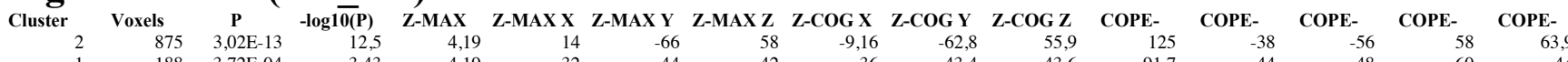

\section{Regressor: Pall(tau $\mathbf{i}=1)$}

\begin{tabular}{|c|c|c|c|c|c|c|c|c|c|c|c|c|c|c|c|}
\hline Cluster & Voxels & $\mathbf{P}$ & $-\log 10(\mathrm{P})$ & Z-MAX & Z-MAX X & Z-MAX Y & Z-MAX Z & Z-COG X & Z-COG Y & Z-COG Z & COPE- & COPE- & COPE- & COPE- & COPE- \\
\hline 10 & 4389 & $1,69 \mathrm{E}-38$ & 37,8 & 5,66 & -54 & -20 & 42 & $-27,9$ & $-11,7$ & 52,7 & 123 & -46 & -40 & 60 & 41 \\
\hline 9 & 2880 & $7,49 \mathrm{E}-29$ & 28,1 & 5,37 & 26 & -48 & -20 & 10,2 & $-74,1$ & $-4,37$ & 94,7 & 2 & -88 & 0 & 38,8 \\
\hline 8 & 697 & $1,17 \mathrm{E}-10$ & 9,93 & 5,25 & -16 & -70 & 52 & $-18,1$ & $-67,7$ & 49 & 94,8 & -16 & -74 & 56 & 45,4 \\
\hline 7 & 537 & $8,17 \mathrm{E}-09$ & 8,09 & 5,87 & -44 & -66 & -18 & $-43,5$ & $-61,2$ & $-18,4$ & 74,6 & -46 & -70 & -20 & 39,1 \\
\hline 6 & 427 & $1,79 \mathrm{E}-07$ & 6,75 & 4,9 & -14 & 14 & 0 & $-18,1$ & 10,7 & 0,875 & 25,9 & -16 & 12 & 0 & 18,2 \\
\hline 5 & 374 & $9,54 \mathrm{E}-07$ & 6,02 & 4,68 & 14 & 16 & 2 & 18 & 11,9 & 2,72 & 24,9 & 20 & 10 & -8 & 18 \\
\hline 4 & 248 & $6,61 \mathrm{E}-05$ & 4,18 & 4,14 & -40 & 36 & 26 & $-38,7$ & 37,1 & 23 & 41,1 & -42 & 40 & 14 & 28 \\
\hline 3 & 209 & $2,79 \mathrm{E}-04$ & 3,55 & 4,61 & 52 & -76 & 4 & 48,6 & $-79,6$ & $-0,197$ & 49,8 & 42 & -88 & -10 & 32 \\
\hline 2 & 122 & $1,01 \mathrm{E}-02$ & 2 & 4,82 & -44 & -2 & 6 & -43 & $-0,926$ & 8,51 & 33,7 & -44 & -2 & 8 & 22,1 \\
\hline 1 & 89 & $4,80 \mathrm{E}-02$ & 1,32 & 4,14 & 32 & 42 & 22 & 33,7 & 43,6 & 23,5 & 33,3 & 32 & 42 & 26 & 26,1 \\
\hline
\end{tabular}

\section{fMRI Feedback Model}

\section{Regressor: Win}

$\begin{array}{rrrr}\text { Cluster } & \text { Voxels } & \text { P } & \text { - } \log \mathbf{1 0}(\mathbf{P}) \\ 10 & 4265 & 1,25 \mathrm{E}-37 & 36,9 \\ 9 & 3327 & 9,47 \mathrm{E}-32 & 3 \\ 8 & 482 & 5,96 \mathrm{E}-08 & 7,22 \\ 7 & 422 & 2,38 \mathrm{E}-07 & 6,6 \\ 6 & 413 & 2,98 \mathrm{E}-07 & 6,53 \\ 5 & 232 & 1,23 \mathrm{E}-04 & 3,9 \\ 4 & 159 & 2,09 \mathrm{E}-03 & 2,68 \\ 3 & 98 & 3,15 \mathrm{E}-02 & 1,5 \\ 2 & 93 & 4,01 \mathrm{E}-02 & 1,4 \\ 1 & 90 & 4,64 \mathrm{E}-02 & 1,3\end{array}$

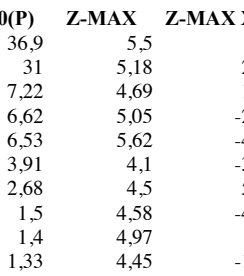

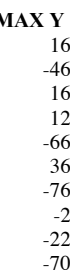

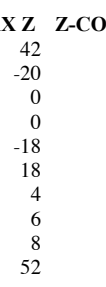

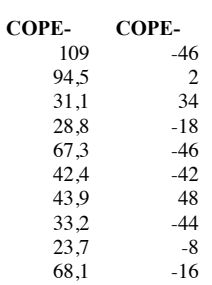

\begin{tabular}{rrr} 
COPE- & COPE- & \multicolumn{1}{c}{ COPE- } \\
-40 & 60 & 39,6 \\
-88 & 0 & 37,3 \\
20 & 8 & 19,4 \\
12 & 0 & 19,6 \\
-70 & -20 & 38,2 \\
40 & 14 & 28,9 \\
-80 & 8 & 29,6 \\
-2 & 8 & 22,2 \\
-22 & 8 & 16,5 \\
-72 & 54 & 44
\end{tabular}

Regressor: PE_Pall[A]

$\begin{array}{rrrr}\text { Cluster } & \text { Voxels } & \mathbf{P}- & -\log 10(\mathbf{P}) \\ & 337 & 2,38 \mathrm{E}-06 & 5,62\end{array}$

Regressor: PE_Pall[nA]

\begin{tabular}{|c|c|c|c|c|c|c|c|c|c|c|c|c|c|c|c|}
\hline Cluster & Voxels & $\mathbf{P}$ & $-\log 10(P)$ & Z-MAX & Z-MAX X & Z-MAX Y & Z-MAX Z & Z-COG X & Z-COG Y & Z-COG Z & COPE- & COPE- & COPE- & COPE- & COPE- \\
\hline 18 & 2597 & $1,20 \mathrm{E}-25$ & 24,9 & 4,84 & 2 & 20 & 50 & $-4,71$ & 14,4 & 49,9 & 99,6 & -32 & -4 & 70 & 34,8 \\
\hline 17 & 1960 & $4,12 \mathrm{E}-21$ & 20,4 & 4,38 & -54 & -22 & 40 & $-40,6$ & $-38,9$ & 49,5 & 126 & -48 & -40 & 60 & 42,6 \\
\hline 16 & 1078 & $6,89 \mathrm{E}-14$ & 13,2 & 5,35 & -16 & 14 & -2 & $-15,1$ & 11,5 & 1,89 & 61,2 & -6 & 2 & 10 & 25,4 \\
\hline 15 & 967 & $7,40 \mathrm{E}-13$ & 12,1 & 5,46 & 16 & 18 & 10 & 14,2 & 8,16 & 5,07 & 50,3 & 8 & 6 & 4 & 22,7 \\
\hline 14 & 465 & 1,19E-07 & 6,92 & 3,99 & 26 & -76 & -16 & 15,8 & $-77,5$ & -12 & 67,4 & 2 & -88 & -4 & 40,4 \\
\hline 13 & 327 & $8,52 \mathrm{E}-06$ & 5,07 & 4,11 & 26 & -54 & -24 & 29,6 & $-51,9$ & $-26,7$ & 31,7 & 32 & -52 & -20 & 18,4 \\
\hline
\end{tabular}




$\begin{array}{rrrrr}12 & 266 & 6,13 \mathrm{E}-05 & 4,21 & 3,99 \\ 11 & 262 & 7,00 \mathrm{E}-05 & 4,15 & 4,04 \\ 10 & 256 & 8,58 \mathrm{E}-05 & 4,07 & 4,25 \\ 9 & 253 & 9,50 \mathrm{E}-05 & 4,02 & 4,11 \\ 8 & 224 & 2,60 \mathrm{E}-04 & 3,58 & 4,12 \\ 7 & 217 & 3,34 \mathrm{E}-04 & 3,48 & 4,29 \\ 6 & 134 & 8,21 \mathrm{E}-03 & 2,09 & 4,2 \\ 5 & 133 & 8,57 \mathrm{E}-03 & 2,07 & 4,04 \\ 4 & 116 & 1,78 \mathrm{E}-02 & 1,75 & 3,96 \\ 3 & 110 & 2,31 \mathrm{E}-02 & 1,64 & 4,07 \\ 2 & 107 & 2,64 \mathrm{E}-02 & 1,58 & 3,77 \\ 1 & 95 & 4,56 \mathrm{E}-02 & 1,34 & 4,09\end{array}$

$\begin{array}{rrr}-54 & 6 & 14 \\ 50 & -68 & 0 \\ -42 & 54 & 12 \\ 36 & -4 & 52 \\ -14 & -22 & 8 \\ 58 & 14 & 4 \\ 30 & -38 & 42 \\ -32 & -66 & -24 \\ 2 & -58 & -26 \\ 30 & -76 & -50 \\ 10 & -104 & 4 \\ 40 & 50 & 14\end{array}$

$-51,5$
51
$-40,4$
33,4
$-10,2$
54,6
35,9
$-32,3$
3,12
21,4
16
41,8

$\begin{array}{rr}9,51 & 21,5 \\ -66,2 & -2,78 \\ 49,7 & 10,2 \\ -4,22 & 56,5 \\ -19,2 & 6,04 \\ 10,5 & 14,8 \\ -41,7 & 52,8 \\ -57 & -25,2 \\ -61 & -29,2 \\ -75,5 & -49,4 \\ -101 & 2,83 \\ 48,3 & 16,5\end{array}$

$\begin{array}{rr}64,3 & -54 \\ 44,3 & 52 \\ 53 & -42 \\ 75,1 & 32 \\ 31,1 & -2 \\ 48,7 & 60 \\ 58,6 & 44 \\ 34,8 & -34 \\ 20,5 & 4 \\ 28,9 & 30 \\ 56,9 & 12 \\ 37,7 & 42\end{array}$

$\begin{array}{rr}18 & -4 \\ -66 & 0 \\ 54 & 12 \\ 0 & 66 \\ -12 & 10 \\ 14 & 2 \\ -40 & 62 \\ -68 & -22 \\ -68 & -38 \\ -76 & -50 \\ -102 & 0 \\ 52 & 12\end{array}$

\section{fMRI Feedback PPI seed IPS}

\section{Regressor: PPI [A > nA]}

$\begin{array}{rrrrr}10 & 4265 & 1,25 \mathrm{E}-37 & 36,9 & 5,5 \\ 9 & 3327 & 9,47 \mathrm{E}-32 & 31 & 5,18 \\ 8 & 482 & 5,96 \mathrm{E}-08 & 7,22 & 4,69 \\ 7 & 422 & 2,38 \mathrm{E}-07 & 6,62 & 5,05 \\ 6 & 413 & 2,98 \mathrm{E}-07 & 6,53 & 5,62 \\ 5 & 232 & 1,23 \mathrm{E}-04 & 3,91 & 4,1 \\ 4 & 159 & 2,09 \mathrm{E}-03 & 2,68 & 4,5 \\ 3 & 98 & 3,15 \mathrm{E}-02 & 1,5 & 4,58 \\ 2 & 93 & 4,01 \mathrm{E}-02 & 1,4 & 4,97 \\ 1 & 90 & 4,64 \mathrm{E}-02 & 1,33 & 4,45\end{array}$


Supplementary Table 3

fMRI behavioral

Models

\section{MODEL: Logit}

beta0

beta1 PV

beta2 Rw

beta3 PV*AM

beta4 Rw*AM

beta5 AM

beta0t TMS

beta1t PV*TMS

beta2t Rw*TMS

beta3t PV*AM*TMS

beta4t Rw*AM*TMS

beta5t AM*TMS

deviance

$\begin{array}{rrrr}\text { Lower95 } & \text { Median } & \text { Upper95 } & \text { Mean } \\ -6,992 & -5,433 & -3,686 & -5,433 \\ 5,898 & 7,817 & 9,776 & 7,834 \\ 1,602 & 3,499 & 5,441 & 3,512 \\ -8,372 & -5,347 & -2,419 & -5,335 \\ -2,842 & -0,142 & 2,908 & -0,073 \\ -0,799 & 2,074 & 4,580 & 2,008 \\ -1,937 & -0,345 & 1,227 & -0,391 \\ -0,906 & 1,000 & 2,891 & 1,011 \\ -2,149 & -0,586 & 1,040 & -0,561 \\ -7,412 & -3,238 & -0,137 & -3,335 \\ -3,105 & 0,406 & 4,203 & 0,325 \\ -1,691 & 1,794 & 5,021 & 1,933 \\ 2.783,320 & 2.829,205 & 2.878,410 & 2.830,358\end{array}$

\begin{tabular}{rr}
\multicolumn{2}{c}{ Mode psr } \\
0,855 & $-5,524$ \\
0,991 & 7,843 \\
0,984 & 3,449 \\
1,536 & $-5,472$ \\
1,520 & $-0,189$ \\
1,424 & 2,102 \\
0,869 & $-0,285$ \\
0,991 & 0,938 \\
0,855 & $-0,787$ \\
1,884 & $-2,958$ \\
1,976 & 0,157 \\
1,848 & 1,349 \\
24,445 & $2,824,596$
\end{tabular}

$24,445 \quad 2.824,596$
pMCMC

$\begin{array}{ll}1,036 & 0,000 * * * \\ 1,026 & 0,000^{* * *} \\ 1,016 & 0,000^{* * *} \\ 1,034 & 0,000^{* * *} \\ 1,023 & 0,468 \\ 1,036 & 0,092 \\ 1,086 & 0,351 \\ 1,077 & 0,155 \\ 1,060 & 0,269 \\ 1,061 & 0,027 * \\ 1,035 & 0,434 \\ 1,056 & 0,150 \\ 1,000 & \end{array}$

MODEL: tau_i

\begin{tabular}{|c|c|c|c|c|c|c|c|c|}
\hline & Lower95 & Median & Upper95 & Mean & SD & Mode & & $\begin{array}{l}\text { pMCMC for } \\
\text { TMS effects }\end{array}$ \\
\hline tau_i & 0,342 & 0,718 & 1,163 & 0,737 & 0,213 & 0,697 & 1,003 & \\
\hline tau_i $*$ TMS & 0,070 & 0,484 & 0,885 & 0,497 & 0,206 & 0,481 & 1,003 & $0,008 * *$ \\
\hline alpha & 0,109 & 3,476 & 12,624 & 4,630 & 3,930 & 1,733 & 1,001 & \\
\hline alpha*TMS & $-4,434$ & $-0,717$ & 3,051 & $-0,706$ & 1,942 & $-0,473$ & 1,040 & 0,688 \\
\hline gamma & 1,948 & 2,947 & 4,007 & 2,972 & 0,535 & 2,871 & 1,002 & \\
\hline gamma * TMS & $-0,233$ & 0,043 & 0,495 & 0,061 & 0,167 & 0,032 & 1,005 & 0,660 \\
\hline beta_0 & $-0,213$ & $-0,036$ & 0,124 & $-0,037$ & 0,086 & $-0,045$ & 1,000 & \\
\hline beta_0* TMS & $-0,249$ & $-0,044$ & 0,154 & $-0,043$ & 0,105 & $-0,055$ & 1,000 & 0,670 \\
\hline beta_1 & 3,871 & 8,255 & 12,741 & 8,274 & 2,284 & 8,203 & 1,002 & \\
\hline beta_1 $*$ TMS & $-0,318$ & 2,728 & 5,461 & 2,745 & 1,430 & 2,696 & 1,007 & 0,054 \\
\hline deviance & $2.866,900$ & $2.902,355$ & $2.935,600$ & $2.902,180$ & 17,902 & $2.902,536$ & 1,003 & \\
\hline
\end{tabular}

MODEL: tau_i + tau_b

\begin{tabular}{|c|c|c|c|c|c|c|c|c|}
\hline & Lower95 & Median & Upper95 & Mean & SD & Mode & & $\begin{array}{l}\text { pMCMC for } \\
\text { TMS effects }\end{array}$ \\
\hline tau_i & 0,249 & 0,583 & 0,979 & 0,595 & 0,186 & 0,542 & 1,001 & \\
\hline tau_i * TMS & 0,141 & 0,491 & 0,864 & 0,507 & 0,187 & 0,468 & 1,000 & $0,001 * * *$ \\
\hline tau_b & 0,292 & 0,400 & 0,503 & 0,400 & 0,053 & 0,405 & 1,003 & \\
\hline tau_b* TMS & $-0,020$ & 0,069 & 0,157 & 0,071 & 0,045 & 0,067 & 1,006 & 0,105 \\
\hline alpha & 0,816 & 1,002 & 1,193 & 1,000 & 0,117 & 1,097 & 1,000 & \\
\hline alpha*TMS & 1,932 & 2,974 & 4,131 & 3,008 & 0,563 & 2,869 & 1,001 & 0,945 \\
\hline gamma & $-0,497$ & 2,461 & 5,016 & 2,527 & 1,375 & 2,442 & 1,006 & \\
\hline gamma * TMS & $-0,381$ & $-0,127$ & 0,126 & $-0,125$ & 0,128 & $-0,126$ & 1,001 & 0,720 \\
\hline beta_0 & $-0,347$ & 0,046 & 0,464 & 0,054 & 0,201 & 0,035 & 1,007 & \\
\hline beta_0* TMS & $-1,738$ & $-0,085$ & 1,876 & $-0,036$ & 1,001 & $-0,011$ & 1,005 & 0,314 \\
\hline beta_1 & 3,563 & 8,024 & 12,672 & 8,069 & 2,318 & 7,716 & 1,002 & \\
\hline beta_1 * TMS & $2.855,570$ & $2.891,910$ & $2.929,850$ & $2.892,347$ & 19,509 & $2.891,273$ & 1,002 & 0,063 \\
\hline deviance & $-0,113$ & 0,093 & 0,302 & 0,093 & 0,106 & 0,081 & 1,001 & \\
\hline
\end{tabular}

MODEL: tau_i + tau_b
tau_i
Lower95 Median Upper95 Mean SD
$0,2088^{\text {Mode }} 0,706{ }^{\text {psrf }} 1,021$
pMCMC for TMS effects 


$\begin{array}{lrrrrrrrr}\text { tau_i * TMS } & 0,017 & 0,510 & 1,033 & 0,516 & 0,256 & 0,510 & 1,008 & 0,009 * * \\ \text { alpha } & 0,108 & 1,023 & 1,898 & 1,031 & 0,542 & 1,118 & 1,000 & 0,076 \\ \text { alpha*TMS } & -1,816 & -0,041 & 1,841 & -0,009 & 1,045 & -0,128 & 1,041 & 0,976 \\ \text { gamma } & 1,659 & 2,677 & 3,743 & 2,703 & 0,528 & 2,611 & 1,102 & 1,000 \\ \text { gamma * TMS } & -0,469 & -0,013 & 0,430 & -0,005 & 0,222 & -0,027 & 1,032 & 1,000 \\ \text { beta_0 } & -0,203 & -0,020 & 0,156 & -0,019 & 0,092 & -0,022 & 1,006 & 0,597 \\ \text { beta_0* TMS } & -0,291 & -0,054 & 0,148 & -0,057 & 0,112 & -0,061 & 1,006 & 0,040 \\ \text { beta_1 } & 5,404 & 9,172 & 13,568 & 9,198 & 2,064 & 9,343 & 1,040 \\ \text { beta_1 * TMS } & 0,297 & 2,463 & 4,933 & 2,483 & 1,169 & 2,423 & 1,014 & 0,037 * \\ \text { Wr } & -0,483 & -0,164 & 0,162 & -0,166 & 0,161 & -0,163 & 1,003 & \\ \text { Wr*TMS } & -0,398 & -0,029 & 0,234 & -0,039 & 0,160 & -0,003 & 1,012 & 0,846 \\ \text { deviance } & 2.776,530 & 2.813,050 & 2.854,830 & 2.814,659 & 20,656 & 2.810,781 & 1,041 & \end{array}$




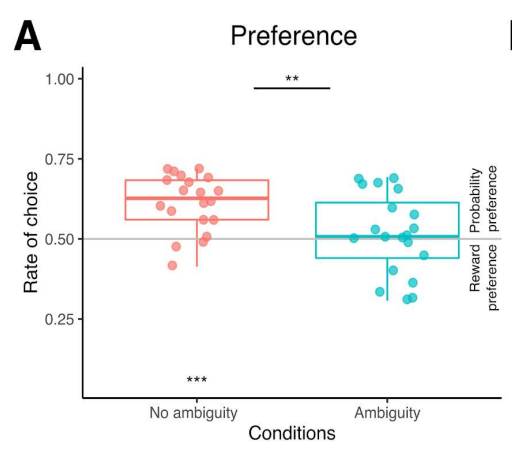

B Preference per ambiguity degree

C Model Comparison
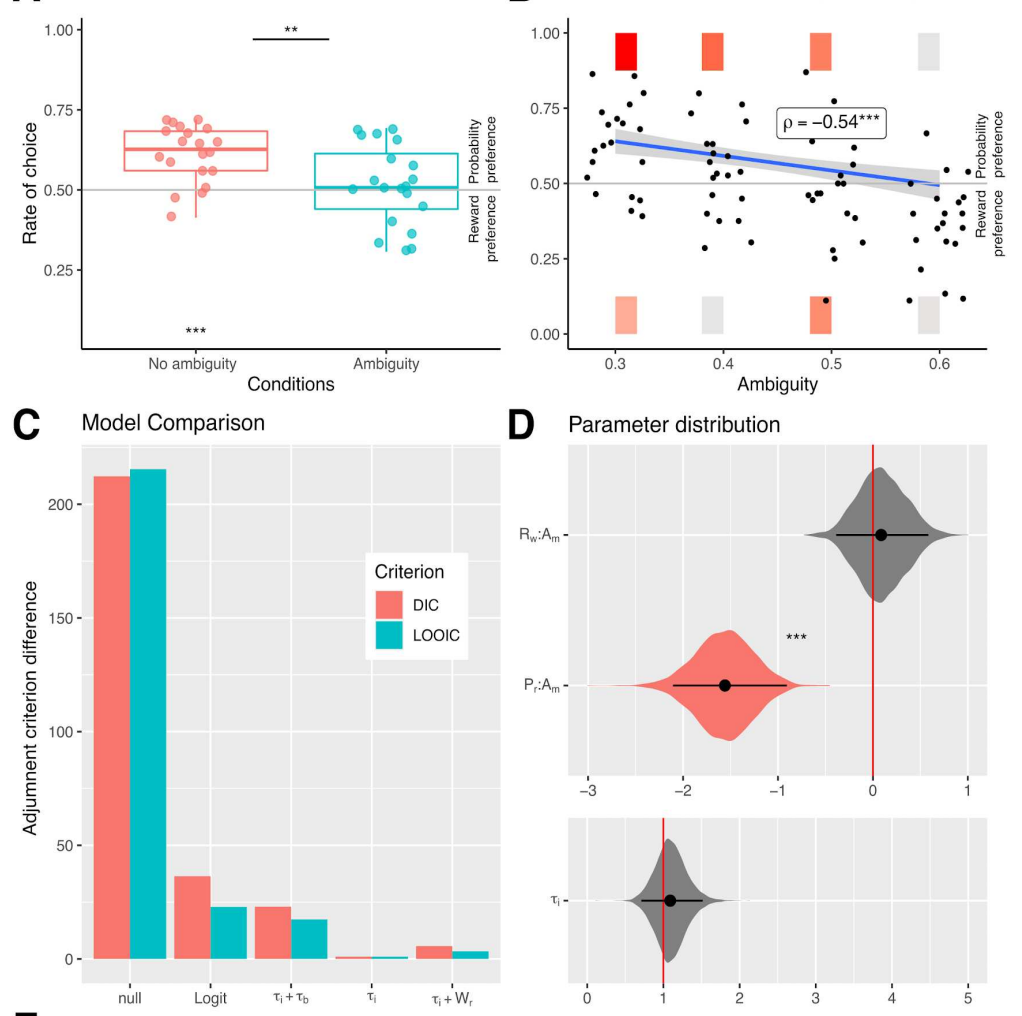

D Parameter distribution
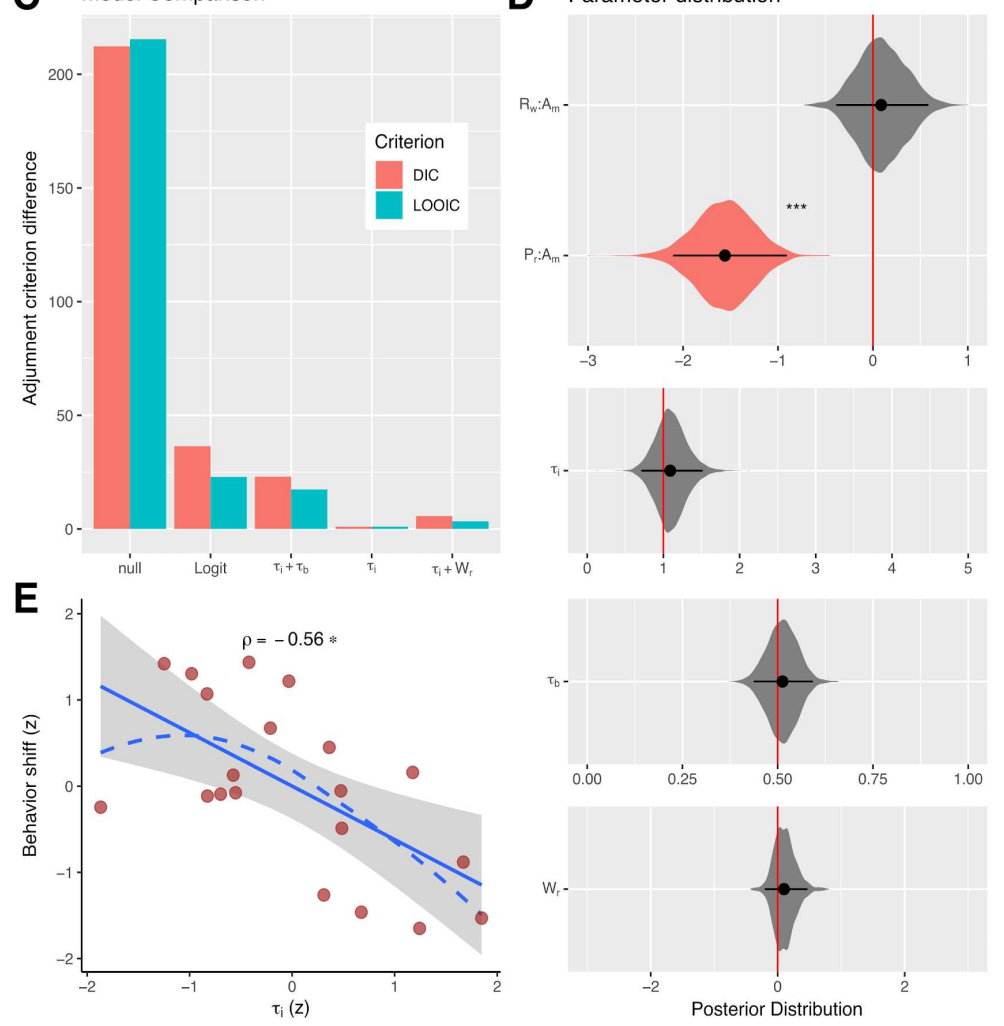

Supplementary Figure 1. Behavioral Results for replication sample. A. Rate of choice where individuals preferred the highest probability per conditions. B. Rate of choice where subjects preferred the highest probability per degree of ambiguity. Black dots represent the rate per individual. Color rectangles indicate the number of individual decisions; red represents the maximum, and light gray, represents the minimum account. The blue line represents the linear regression, and the gray area is the standard error. $\mathbf{C}$ Model fitting comparison using DIC, red, and LOOIC, green. D Posterior distribution of the key parameters for each model. Black dots represent the mean of the distribution and black lines the $95 \%$ high-density intervals. The colored areas represent the complete posterior distribution. E Correlation between decision shift (difference 
between the rate of choices that subject prefers the highest probability between condition, ambiguity less no-ambiguity) and $\tau_{i}$ parameters. Red dots represent each subject. The solid blue line represents the linear regression, the dotted blue line the LOESS regression, and the gray area represents the standard error. $*$ indicates $\mathrm{p}<0.05, * * \mathrm{p}<0.01, * * * \mathrm{p}<0.001$.

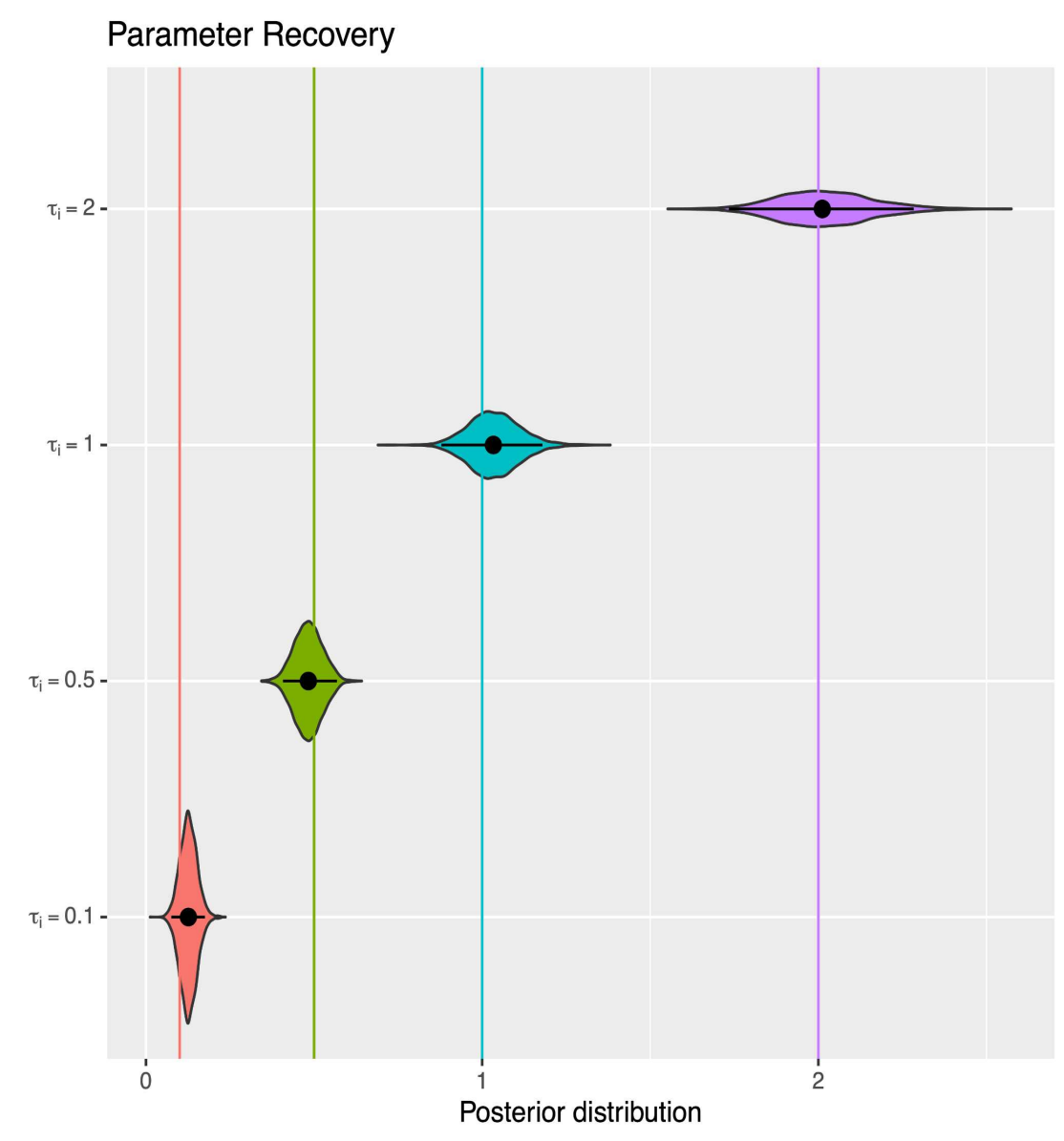

Supplementary Figure 2. Posterior distribution of tau parameters recovery from simulated dated generated by different tau parameters. For the simulation, all the other model parameters were fixed using the mean of the posterior distribution fitted from the real data. 
KEY RESOURCES TABLE

\begin{tabular}{|c|c|c|}
\hline REAGENT or RESOURCE & SOURCE & IDENTIFIER \\
\hline \multicolumn{3}{|l|}{ Software and Algorithms } \\
\hline MATLAB 2015b & MathWorks & $\begin{array}{l}\text { RRID: SCR_001622 } \\
\text { https://www.mathwork } \\
\underline{\text { s.com }}\end{array}$ \\
\hline R Project for Statistical Computing & R Core Team, 2016 & $\begin{array}{l}\text { RRID:SCR_001905 } \\
\text { http://www.r- } \\
\text { project.org/ }\end{array}$ \\
\hline Brainstorm 3 & & RRID: SCR_001761 \\
\hline LAN toolbox & In-house scripts & $\begin{array}{l}\text { RRID: SCR_017629 } \\
\text { https://github.com/neur } \\
\underline{\text { ocics/LAN_current }}\end{array}$ \\
\hline FSL & FSL 5.0.10 & $\begin{array}{l}\text { RRID: SCR_002823 } \\
\text { http://www.fmrib.ox.a } \\
\text { c.uk/fs1/ }\end{array}$ \\
\hline Presentation & $\begin{array}{l}\text { Neurobehavioral } \\
\text { system }\end{array}$ & $\begin{array}{l}\text { RRID: SCR_00252 } \\
\underline{\text { http://www.neurobs.co }} \\
\underline{\mathrm{m} /}\end{array}$ \\
\hline DUneuro software & & http://duneuro.org/ \\
\hline SimNIBS tool & & $\begin{array}{l}\text { RRID: SCR_014109 } \\
\text { https://simnibs.github.i }\end{array}$ \\
\hline
\end{tabular}




\begin{tabular}{|l|l|l|}
\hline & & $\begin{array}{l}\text { o/simnibs/build/html/i } \\
\text { ndex.html }\end{array}$ \\
\hline SMP12 & & $\begin{array}{l}\text { RRID: SCR_007037 } \\
\text { https://www.fil.ion.ucl. } \\
\text { ac.uk/spm/software/sp } \\
\text { m12/ }\end{array}$ \\
\hline
\end{tabular}

Further information and requests for resources should be directed to the Lead Contact, Pablo Billeke (pbilleke@udd.cl) 


\section{Supplementary Files}

This is a list of supplementary files associated with this preprint. Click to download.

- ValdebenitoOyarzoSI08022022.pdf 